\title{
1 Skin inflammation driven by differentiation of quiescent tissue-resident ILCs into a
} 2 spectrum of pathogenic effectors

3

4 Piotr Bielecki*,1, Samantha J. Riesenfeld*,2, Monika S. Kowalczyk*,2, Maria C. Amezcua 5 Vesely $^{1,3}$, Lina Kroehling ${ }^{1}$, Parastou Yaghoubi ${ }^{1}$, Danielle Dionne ${ }^{2}$, Abigail Jarret ${ }^{1}$, Holly R. 6 Steach $^{1}$, Heather M. McGee ${ }^{1,4}$, Caroline B. M. Porter ${ }^{2}$, Paula Licona-Limon ${ }^{1,5}$, Will Bailis ${ }^{1}$, 7 Ruaidhri P. Jackson ${ }^{1}$, Nicola Gagliani ${ }^{1,6,7,8}$, Richard M. Locksley ${ }^{3,9}$ Aviv Regev $^{\wedge, 2,3,10}$, Richard A. $8 \quad$ Flavel1 ${ }^{\wedge 1,3}$

$10{ }^{*}$ These co-first authors contributed equally to this work

$11 \quad{ }^{1}$ Department of Immunobiology, Yale University School of Medicine, New Haven, CT 06520, USA

$12 \quad{ }^{2}$ Klarman Cell Observatory, Broad Institute of MIT and Harvard, Cambridge MA 02142

$13{ }^{3}$ Howard Hughes Medical Institute

$14{ }^{4}$ Department of Radiation Oncology, Icahn School of Medicine at Mount Sinai, New York, NY 10029

$15{ }^{5}$ Departamento de Biología Celular y del Desarrollo, Instituto de Fisiología Celular, Universidad Nacional Autónoma 16 de México, México City 04510

$17{ }^{6}$ Department of General, Visceral and Thoracic Surgery, University Medical Center Hamburg-Eppendorf, 20246

18 Hamburg, Germany;

$19{ }^{7}$ I. Department of Medicine, University Medical Center Hamburg-Eppendorf Hamburg-Eppendorf, 20246 Hamburg,

20 Germany;

$21{ }^{8}$ Immunology and Allergy Unit, Department of Medicine Solna, Karolinska Institute, 17176 Stockholm, Sweden

$22{ }^{9}$ Department of Medicine, University of California San Francisco, San Francisco, CA, USA

$23{ }^{10}$ Koch Institute of Integrative Cancer Research, Department of Biology, Massachusetts Institute of Technology,

24 Cambridge, MA 02140

$25 \wedge$ Correspondence to: A.R aregev@broadinstitute.com and R.A.F richard.flavell@yale.edu 
26 Psoriasis pathology is driven by the type 3 cytokines IL-17 and Il-22, but little is understood

27 about the dynamics that initiate alterations in tissue homeostasis. Here, we use mouse models, single-cell RNA-seq (scRNA-seq), computational inference and cell lineage mapping to show that psoriasis induction reconfigures the functionality of skin-resident ILCs to initiate disease. Tissue-resident ILCs amplified an initial IL-23 trigger and were sufficient, without circulatory ILCs, to drive pathology, indicating that ILC tissue remodeling initiates psoriasis. Skin ILCs expressed type 2 cytokines IL-5 and IL-13 in steady state, but were epigenetically poised to become ILC3-like cells. ScRNA-seq profiles of ILCs from psoriatic and naïve skin of wild type (WT) and $\operatorname{Rag} 1^{-/-}$mice form a dense continuum, consistent with this model of fluid ILC states. We inferred biological "topics" underlying these states and their relative importance in each cell with a generative model of latent Dirichlet allocation, showing that ILCs from untreated skin span a spectrum of states, including a naïve/quiescent-like state and one expressing the $C d 74$ and $I l 13$ but little $I l 5$. Upon disease induction, this spectrum shifts, giving rise to a greater proportion of classical II5- and Ill3expressing "ILC2s" and a new, mixed ILC2/ILC3-like subset, expressing II13, II17, and Il22. Using these key topics, we related the cells through transitions, revealing a quiescence-ILC2ILC3s state trajectory. We demonstrated this plasticity in vivo, combining an IL-5 fate mouse with IL-17A and IL-22 reporters, validating the transition of IL-5-producing ILC2s to IL22- and IL-17A-producing cells during disease initiation. Thus, steady-state skin ILCs are actively repressed and cued for a plastic, type 2 response, which, upon induction, morphs into a type 3 response that drives psoriasis. This suggests a general model where specific

47 immune activities are primed in healthy tissue, dynamically adapt to provocations, and left unchecked, drive pathological remodeling. 


\section{INTRODUCTION}

50 Psoriasis pathology is driven by the type 3 cytokines IL-17 and Il-22 (Nograles, Zaba et al. 2008,

51 Cai, Shen et al. 2011). The dermal inflammation and acanthosis induced by IL-23 are thought to

52 be mediated by the Th17-cell associated cytokine IL-22. While both IL-17 and IL-22 are produced

53 at elevated levels in psoriatic skin, the major cell source of these cytokines during disease remains

54 unclear. In psoriasis patients, $\gamma \delta$ T cells were found to be greatly increased in affected skin and

55 produced large amounts of IL-17, suggesting they may play a key role in pathogenesis (Cai, Shen

56 et al. 2011). Recently, however, IL-17 and IL-22 producing ILC3s have been proposed to be a

57 significant source of cytokine production during psoriasis (Teunissen, Munneke et al. 2014,

58 Villanova, Flutter et al. 2014). These cells are thought to be activated in response to IL-1 $\beta$ and IL-

5923 , whose levels correlate with disease severity, and are decreased following antitumor necrosis

60 factor- $\alpha($ anti-TNF $\alpha)$ treatment. The presence of a novel ILC population in psoriatic skin that

61 responds to one of the most effective biologic therapeutics suggests that dysregulation of ILCs is

62 a contributing factor to psoriasis pathogenesis. While ILC3s dominate psoriatic skin (Pantelyushin,

63 Haak et al. 2012), in healthy individuals the majority of ILCs are represented by group 2 ILCs,

64 defined by IL-5 and IL-13 production (Roediger, Kyle et al. 2013, Spencer, Wilhelm et al. 2014).

65 We wanted to determine at what point during disease progression the frequency of ILC2s and

66 ILC3s shifts and whether a potential for conversion between these cell types underlies this disease.

67

68 High parallel single-cell RNA sequencing (scRNA-seq) has become a powerful tool for unbiased analysis of various cells types. Analyses of immune cell classes, such as ILCs, typically treat them

70 as collections of discrete immune cell "types", yet these cell types may share important biological

71 signals and have been observed in some contexts to essentially continuously span a functional 
72 spectrum. To capture and explore fluid, mixed transcriptional states, we used latent Dirichlet

73 allocation (LDA), or "topic modeling", a statistical data mining approach for discovering the

74 abstract topics that explain the words occurring in a collection of text documents (Blei 2003).

75 Applied to scRNA-Seq, each "document" corresponds to a cell, and a "topic" corresponds to a

76 biological program, modeled as a distribution over expressed genes, rather than words. Given the

77 number of topics as a parameter, both topics and the mixture weights in cells are inferred without

78 supervision. LDA was independently introduced in population genetics to model admixed

79 individuals with ancestry from multiple populations (Pritchard, Stephens et al. 2000). In genomics,

80 it has been applied to deconvolute cell types in population RNA-seq (Repsilber, Kern et al. 2010,

81 Schwartz and Shackney 2010, Shen-Orr, Tibshirani et al. 2010, Ahn, Yuan et al. 2013, Lindsay

82 2013, Quon, Haider et al. 2013, Wang, Gong et al. 2015), and proposed for finding structure in

83 bulk or single-cell RNA-seq, for example, in inference of confounding batch effects (Dey, Hsiao

84 et al. 2017).

86 RESULTS

87 To determine which cells are key to initiate psoriatic disease, we studied a subcutaneous IL-23

88 injection model, which leads to increased skin thickness after five days of daily injections (Fig.

89 1A, Fig. S1A). First, we assessed the role of different immune cell types in this model (Fig. 1B,

90 Fig. S1B and C). Consistent with previous results, the $\mathrm{Rag}^{-/-} \mathrm{Il} 2 \mathrm{rg}^{-/-}$double mutant, which lacks

91 all lymphocytes, did not show any increase in ear thickness, whereas $\operatorname{Rag} 1^{-/-}$mice, which have

92 intact ILCs, showed significant increase in skin thickness over the treatment course. This is also

93 consistent with an increased number of human ILC3s recently observed in psoriatic patients

94 (Teunissen, Munneke et al. 2014, Villanova, Flutter et al. 2014). Moreover, while $\gamma \delta$ T cells have 
95 been implicated in a longer treatment course (Cai, Shen et al. 2011, Pantelyushin, Haak et al.

96 2012), analyzing $\mathrm{Tcrd}^{-/-}$mice, which lack only $\gamma \delta \mathrm{T}$ cells, we found no evidence that they contribute

97 to disease initiation (Fig. 1B). Next, to further confirm the role of ILCs in disease initiation, we

98 adoptively transferred sorted skin ILCs from untreated WT mice into $\mathrm{Rag}^{-/-} \mathrm{Il}_{2 \mathrm{rg}} \mathrm{rl}^{-/}$mice, and

99 observed significant skin thickening in treated versus untreated recipient mice (Fig. 1C). Finally,

100 we assessed the contributions of circulatory versus tissue-resident lymphocytes in the psoriasis

101 model, because recent studies of inflammation in several peripheral tissues suggested different

102 involvement of circulatory and tissue resisdent ILCs (Dyring-Andersen, Geisler et al. 2014,

103 Gasteiger, Fan et al. 2015, Li, Hodgkinson et al. 2016, Yang, Hu et al. 2016, Huang, Mao et al.

104 2018). We compared disease phenotype between control mice and those treated with FTY720,

105 which blocks signaling from the S1P1 receptor, preventing egress of $\mathrm{T}$ cells from secondary

106 lymphoid tissues and limit trafficking of induced ILC2s (Matloubian, Lo et al. 2004, Huang, Mao

107 et al. 2018). FTY720-treated mice had the expected reduction of circulating total white blood cells,

108 but showed no difference in psoriasis phenotype induction upon IL-23 administration compared

109 with untreated controls, in both WT or Ragl $1^{-/-}$(lacking T and B cells) mice (Fig. 1C and E). Thus,

110 in contrast to a model of lung inflammation (Huang, Mao et al. 2018), in psoriasis, tissue-resident

111 ILCs are sufficient to drive disease pathology and are critical for amplifying the response to IL-

11223.

114 We observed that skin ILCs expressed the type 2 cytokines IL-5 and IL-13 in steady state, but

115 showed potential to plastically to assume ILC3-like states. Consistent with prior reports that naïve

116 mouse skin ILCs are comprised almost exclusively of GATA3 ${ }^{+}$ILC2s (Roediger, Kyle et al. 2013),

117 total ILCs isolated from healthy mouse skin and treated with the type 2 alarmin cytokines IL-25 
118

119

120

121

122

123

124

125

126

127

128

129

130

131

132

133

134

135

136

137

138

139

140

and L-33 had a strong type 2 activation, as indicated by expression of Areg and Ill3 (Fig. S1D). However, total ILCs treated with IL-23 and IL-1 $\beta$ instead strongly expressed $I l 22$ and Il17a (Fig. S1D), suggesting that tissue-resident skin ILCs may have potential for type 2-3 plasticity. Such plasticity has been previously reported in IL-17A co-expressing "inflammatory ILC2s" in the lung (Huang, Guo et al. 2015, Zhang, Xu et al. 2017), similar to reported type 3-1 plasticity in gut and tonsil ILCs and type 2-1 plasticity in blood ILCs (Cella, Otero et al. 2010, Bernink, Krabbendam et al. 2015, Bal, Bernink et al. 2016, Lim, Menegatti et al. 2016, Ohne, Silver et al. 2016, Silver, Kearley et al. 2016). Moreover, while inflammation and skin thickness reverted to near-baseline levels within 10 days after the initial IL-23 injection (Fig. 1F), this initial challenge promoted a stronger type 3 response upon re-challenge. Specifically, mice showed a significantly more severe phenotype after a second series of IL-23 injections, compared to their initial response (Fig. 1F and G). This was also observed in mice treated with FTY720 during the primary injection (Fig. S1E), suggesting that the plastic psoriatic response is not due to ILC recruitment.

We hypothesized that this plasticity may be encoded epigenetically. To test this hypothesis, we profiled sorted total skin ILC populations from naïve mice by ATAC-seq. We observed the expected open chromatin signature at the TSS of Gata3, Il5 and Il13 and not at the TSS of Tbx21 or Rorc, which encode T-bet and Roryt, the hallmark transcription factors (TFs) of ILC1s and ILC3s, respectively, or at the TSS of $I l 22, I l 17 a$, or $I l 17 f$ (Fig. S1F and G). In support of our hypothesis, we also observed strong ATAC-seq peaks at at promoters of some type 3 genes in TFs binding sites, such as Batf, Maf, and Irf (Ciofani, Madar et al. 2012, Li, Spolski et al. 2012, Zhong, Cui et al. 2016) (Fig. 1H, Fig. S1G), which are known to regulate Th17 cells. Taken together, our data support a model where IL-23 induces psoriasis by remodeling a heterogeneous, tissue-resident 
141 ILC population with unexpected potential for differentiation, rather than by recruiting circulating

142 ILCs to replace a homogenous, terminally differentiated skin-resident ILC2 population.

144 To assess the molecular heterogeneity of skin-resident ILCs and its functional implications for the

145 IL-23 response, we collected massively parallel scRNA-seq profiles from sorted pure total ILCs

146 from WT and $\mathrm{Ragl}^{-/-}$mice from naïve and IL-23 induced conditions, predominantly uncovering a

147 large heterogeneous population of cells (Fig. 1I). Specifically, clustering on principle components,

148 followed by differential expression analysis (Methods), identified a few discrete subsets of cells, 149 including a $\operatorname{Ragl}^{-/-}$-specific subset $(A)$, a cluster of proliferating cells from all conditions and 150 genotypes $(B)$, and a cluster specific to the induced condition with very high $I l 22$ expression and

151 some $I l 13$ expression (C) (Fig. 1J, Fig. S1H). However, the vast majority of cells (81\%) formed a

152 single, large heterogeneous and continuous "cloud" $(D)$, which was not simply driven by technical

153 factors (Methods), with multiple sub-regions enriched for specific functional programs, including

154 type 2 immune response (Fig. 1J). Importantly, no single partitioning conformed to the expression

155 of key genes and processes, and moreover, some biological processes were unexpectedly shared 156 across subsets of the cells from distinct clusters (Fig. 1J). This highlighted the diversity of potential 157 cell states, and the need to capture them by more nuanced computational analysis.

159 To characterize the heterogeneity of ILCs during IL-23 response, we created a generative topic 160 model based on LDA. Analogous to a text document, a cell is modeled as a mixture of a small 161 number of topics, where the mixture weights indicate the relative prominence of the corresponding 162 biological process in that cell. Multiple topics may include the same gene, reflecting the gene's 163 roles in different processes. Topic modeling permits a cell to have multiple, non-hierarchical 
"identities" that potentially differ in importance, a feature particularly relevant for analyzing cellular plasticity (Fig. 2A). Indeed, we observed complex patterns of topic sharing across clusters, suggesting that topic weights capture relationships not well described by clusters and, through their functional interpretation, enable a more nuanced view of similarities and differences among cells

(Fig. 2B). Several choices for the number of topics may result in valid models, though too large a number of topics can result in overfitting and low interpretability. We found that in this dataset, 15 topics captured important changes during disease induction, as well as other signals, without

171 obvious signs of overfitting (Fig. S2A, Methods).

172

173 Our topics spanned three categories: (1) highly ribosomal- or mitochondrial-dominated (e.g.,

174 Topic 1, 6), possibly reflecting technical quality or cell size, (2) cluster-specific topics (e.g., Topic

$1757,14,15)$, and (3) "sub-regional" topics, that is, those featured in sub-regions of the "cloud", also

176 often simultaneously present in sub-regions of other clusters (e.g., Topics 2, 4, 8, 11, 13) (Fig. 2C

177 and D, Fig. S2B-D). "Cell quality" topics can help distinguish the influence of technical

178 confounders better than simple thresholds, but also may reflect a cell's level of biological 179 activation (Wallrapp, Riesenfeld et al. 2017). "Cluster-specific topics" are analogous to results 180 from standard differential expression analysis. For example, cluster $C$ is unique in having large 181 weights for Topic 15, which is characterized by expression of ILC3-associated genes $\mathrm{Il22}$, Il17a, 182 and $I l 17 f$, as well as the cytotoxic gene Gzmb and the type 2 genes $L y 6 a$ (Sca-1) and $I l 13$ (Fig. 2C

183 and D, Fig. S2B and C). As another example, Topic 7 is uniquely highly weighted in cells from 184 the $\operatorname{Ragl}^{-/-}$-specific cluster $A$, and features the NK-associated genes Klrdl and Tyrobp and the 185 immunoglobulin E receptor Fcerlg, indicating that agg $^{-/-}$mice might have an overrepresentation 186 of skin-resident ILC1s (Fig. 1J, Fig. S2D). 
The "sub-regional" topics highlighted functional states that are prominent within the "cloud" and span across cluster boundaries, showing that ILCs from untreated skin span a spectrum of immune states, including one characterized by $V p s 37 b$ expression (Topic 2), a naïve/quiescent-like state (Topic 8) and an activated state related to antigen presentation (Topic 11). Notably, this may mirror "functional compartmentalization" reported in gut ILCs in homeostasis (Gury-BenAri, Thaiss et classical Il5- and Il13-expressing "ILC2s" (Topic 13), as well as a mixed ILC2/ILC3-like state characterized by strong expression of Il13, Il17, and Il22 (Topic 15) (Fig. 2C and D, Fig. S2B). Specifically, Topic 2, mainly present in the "cloud", distinguishes between the untreated and induced conditions, partly through ribosomal genes that may reflect differences in size between naïve and activated cells (Fig. 2C). Topic 8 is characterized by expression of TFs previously associated with both T- or B-cell quiescence, such as Klf2/Klf4 (Carlson, Endrizzi et al. 2006, Cao, Sun et al. 2010) and Zfp36l2 (Galloway, Saveliev et al. 2016, Salerno, Engels et al. 2018), and with repression of Th17 genetic programs, such as $T s c 22 d 3$ (Yosef, Shalek et al. 2013), and may thus reflect an actively maintained quiescent ILC state (ILC0) (Fig. 2C and D, Fig. S2C). Topic 11, which is present in cells from both WT conditions and the Rag $1^{-/-}$induced condition, features genes associated with antigen presentation, including MHCII invariant chain and MIF receptor Cd74(Schroder 2016) and Cd83 (Kuwano, Prazma et al. 2007), and type 2 ILCs (e.g., Il13, Ccl1, 206 and Dgat2, though not Il5) (Robinette, Fuchs et al. 2015, Gury-BenAri, Thaiss et al. 2016, 207 Wallrapp, Riesenfeld et al. 2017, Ricardo-Gonzalez, Van Dyken et al. 2018) (Fig. 2C and D). 208 Topic 13, highlighting a substantial sub-region of both the "cloud" and induced-specific cluster $C$, 209 is more specific to WT disease induction, uniquely expresses $I l 5$, and also includes other type 2 
genes, such as Cxcl2, Il1rl1 (ST-2), Il13, and Ly6a (Sca-1), the latter of which featured in all

211 induced topics (Fig. 2C and D, Fig. S2B and C). The presence of some cells with high weights

212 for both Topics 13 and 15 indicates that an activated type 2 response apparently co-exists with the

213 anticipated type 3 response. Finally, Topic 4, which is largely mutually exclusive with Topic 13

214 across cells, includes genes involved in actin remodeling, a process previously shown to be

215 important during T-cell activation (Kumari, Curado et al. 2014) (Fig. 2C and D).

217 We hypothesized that cells can transition between some of these programs or states, as such 218 transitions would be consistent with the dense transcriptional continuum observed. Unlike 219 pseudotime inference (Trapnell, Cacchiarelli et al. 2014, Haghverdi, Buttner et al. 2016), topic 220 modeling does not assume the existence of an "axis" of progression, which may not exist in settings

221 such as the untreated condition. Moreover, when a trajectory does exist, it may be reflected only

222 in specific aspects of the transcriptional profiles. Indeed, a temporal "induction" dimension in our

223 data was revealed most clearly when we focused on specific topics related to immune repression

224 or activation. To identify transitional relationships in the context of the biological processes 225 reflected by these topics, we created a diffusion map only from those cells highly weighted for 226 Topics $2,4,8,11,13$, and 15 , but not for Topics 6 or 7 , and used only the most distinguishing genes 227 for each topic as input (Fig. S3A-C, Methods).

229 The diffusion map (Fig. 3A, Fig. S3D) proposes several parallel state transitions that cells undergo 230 in the tissue, in particular highlighting a quiescence-ILC2-ILC3s state trajectory in the disease.

231 First, cells from the naïve condition lie in a triangular region in the plane spanned by diffusion 
and 11 ("antigen presentation"), respectively (Fig. 3A and B.i-iii). Their distribution throughout

234 the triangle suggests that in the untreated condition, cells range over all mixtures of these states.

235 Second, DC1 captures the induced response shared in both WT and Ragl $^{-/-}$mice (Fig. 3A, Fig.

236 S3D), such that as their DC1 coordinate ("induction") increases, cells typically have relatively

237 lower weights for Topics 2, 8, and 11 (Fig. 3B.i-iii, Fig. S3E.i-iii), and higher weights for Topic

23815 (“Il22/Il117”), Topic 4 (“actin remodeling”), and, specifically for cells from WT mice, Topic

23913 ("Il5/Cxcl2") (Fig. 3B.iv-vi, Fig. S3E.iv-vi). Genotype-specific differences in the induction

240 response are further captured by DC4, such that cells from WT and $\operatorname{Rag} 1^{-/-}$mice have increasingly

241 different DC4 coordinates as DC1 coordinate increases (Fig. S3D).

243 A focused diffusion map model (Fig. 3C) generated only from cells up-weighted for Topic 8, 13,

244 or 15 (Methods), shows continuous expression changes from Topic 8 to 13 to 15, as DC 1 (in this

245 map) coordinate increases (Fig. 3D and E). Indeed, DC1 is particularly well correlated with

246 expression of the gene Srgn, a proteoglycan that is critical for the trafficking and storage of Gzmb

247 (Sutton, Brennan et al. 2016), which suggests that expression of this gene could be an early

248 indicator of a trajectory toward type 3 activation, visible before expression of either Gzmb or type

2493 cytokines (Fig. 3E). The expression changes observed across Topic 8, 13, and 15 are consistent

250 with a novel model of immune activation in which a type 3 stimulus (IL-23) causes skin-resident

251 naïve/quiescent ILCs to undergo type 2 activation, followed by transition to ILC3-like cells.

253 Finally, we tested the model's predictions of a quiescent-ILC2-ILC3 trajectory. First, we validated

254 the quiescent state by ATAC-Seq of sorted total skin ILC populations from naïve mice. Consistent 255 with Topic 8 ("naïve-quiescent") highlighted by the scRNA-seq analysis, the loci for the TFs Klf2, 
256 Klf4, previously associated with quiescence (Carlson, Endrizzi et al. 2006, Cao, Sun et al. 2010),

$257 T s c 22 d 3$ and $Z f p 36 l 2$, associated with Th17 genetic program repression (Yosef, Shalek et al. 2013,

258 Galloway, Saveliev et al. 2016, Salerno, Engels et al. 2018) and Cebpb, involved in hematopoiesis

259 (Tsukada, Yoshida et al. 2011), had open chromatin signatures at their TSS (Fig. 4A).

260

261 Next, we tested the prediction of a transition during disease of IL-5-expressing ILC2s into IL-

262 22/IL-17A-expressing ILC3-like cells. We generated an IL-5 fate reporter mouse from IL-5-cre-

263 dTomato (Red5) (Nussbaum, Van Dyken et al. 2013) and Rosa26 flox-Stop-floxYFP, which we then

264 combined with IL-17A ${ }^{\mathrm{GFP}}$ (Esplugues, Huber et al. 2011) and IL-22 ${ }^{\mathrm{BFP}}$ expression reporters (Fig.

265 4B). Consistent with our model, after IL-23 injection, 10\% of the IL-22- and IL-17A-expressing

266 cells were indeed ex-IL-5 producing cells, as measured by fate mapping of ILC2s, and a second

267 IL-23 challenge further elevated the number of ex-IL-5 cells producing IL-22 and IL-17A (Fig.

268 4C and D). Moreover, cells that expressed ILC3 type cytokines no longer expressed IL-5 (Fig.

269 4D). Our results show the in vivo potential for plasticity among skin ILCs and demonstrates that

270 some cells expressing ILC3 type cytokines expressed IL-5 at one stage of their lifetime. Finally,

271 we also tested our model's prediction that there is a subset of skin ILCs in the psoriasis model that

272 co-expresses the type 2 cytokine IL-13 with both of the type 3 cytokines IL-22 and IL-17A. Indeed,

273 intracellular measurements of these three cytokines showed that, consistent with the predictions,

274 nearly $20 \%$ in Ragl $^{-/}$and $10 \%$ in WT and Tcrd $^{-/-}$of cells expressing IL-22 and IL-17A also co-

275 express IL-13 (Fig. 4E and F).

276

277

278 


\section{DISCUSSION}

Experimentally combining scRNA-seq, ATAC-seq, and in vivo fate mapping in the psoriasis

281

282

283

284

285

286

287

288

289

290

291

292

293

294

295

296

297

298

299

300

301

mouse model with new analytical approaches, we showed the presence of previously undescribed naïve/quiescent-like tissue-resident ILCs and the ability of activated ILC2s to differentiate to pathological ILC3s. We further discovered a novel subset of ILCs expressing IL-13 and IL-22/IL17A in response to IL-23 stimulation. Our work highlights the limitation of experimental and computational analyses of immune cells that treat them as discrete immune "types", when immune cells may share biological signals and span continuous spectra. In our system, we did not observe any discrete boundaries in single-cell expression profiles that neatly partitioned naïve/quiescentlike ILCs from activated type 2 cells, or type 2 cells from type 3 cells. Rather, the entire population of skin-resident ILCs was functionally reconfigured and its spectrum shifted by disease induction. Indeed, imposing stress on an immune cell population may allow rapid shifting of such a spectrum towards alternative cell fates (Tusi, Wolock et al. 2018), and pathways similar to those we uncovered in the skin may play roles in other tissues. Importantly, this also suggests that studies of ILCs sorted on expression of specific cytokines, such as IL-5 (Ricardo-Gonzalez, Van Dyken et al. 2018), may not have fully assessed this larger continuum. This model substantially revises previous interpretations and can provide a unified framework for some observations in other systems, such as "functional compartmentalization" within ILC types and gut ILCs that could not be readily assigned to a single ILC type (Gury-BenAri, Thaiss et al. 2016). These studies did not report a differentiation from ILC2 to ILC3, (but rather reported that a core ILC2 module was robust to antibiotic perturbation, albeit with increased expression of genes associated in homeostasis with ILC3s (Gury-BenAri, Thaiss et al. 2016), which may reflect tissue-specific differences in ILC features (Ricardo-Gonzalez, Van Dyken et al. 2018). Computational models and biological 
302 interpretations that allow for such fluidity, including topic modeling, are thus valuable for

303 uncovering biological phenomena because they highlight signals, such as, in our case, type 2

304 activation, shared by cells in distinct clusters, and reveal drivers of heterogeneity among cells

305 within a single group, such as the ILC “cloud". This type of presentation is consistent with recent

306 studies of HSCs, where individual precursors have probabilistic fate maps, tilted towards but not

307 commited to specific outcomes (Carrelha, Meng et al. 2018, Laurenti and Gottgens 2018). Such

308 approaches should be valuable in uncovering how tissue-resident ILCs, and other cell types, may

309 globally respond to a stimulus, and undergo dynamic, plastic activation to reach the necessary state

310 for shaping the tissue landscape. 
METHODS

313 Mice. C57BL/6, Tcrd $^{-/-}$and Rosa26 flox-stop-floxYFP Ai3(RCL-EYFP) mice were purchased from the 314 Jackson Laboratories. Rag ${ }^{-/-}$and $\mathrm{Rag}^{-/-} \mathrm{IL}_{\mathrm{Lg}} \mathrm{rg}^{-/}$were purchased from Taconic Biosciences. IL-5

315 Cre, dTomato (Red5/R5) from Dr Locksley laboratory. The IL-5 fate reporter in this work was

316 generated by crossing Red5 with Ai3(RCL-EYFP) with IL-17A ${ }^{\mathrm{GFP}}$ (Esplugues, Huber et al. 2011)

317 and IL-22 $2^{\mathrm{BFP}}$ generated in our laboratory. In order to maximize the Cre recombination and increase

318 the signal of Rosa $26^{\text {YFP }}$ positive cells, we used homozygous IL-5 $5^{\text {dTomato,Cre }}$. We observed little to 319 no difference in IL-23 induced skin thicknening (Fig. S4A).

321 All mice were kept under specific pathogen-free (SPF) conditions in the animal facility at Yale

322 University. Age- and sex-matched littermates between 10 to 14 weeks of age were used for all 323 experiments. Unless with special instructions, mice were randomly assigned to different 324 experimental groups and each cage contained animals of all different experimental groups. Both 325 male and female mice were used in experiments. Animal procedures were approved by the 326 Institutional Animal Care and Use Committee (IACUC) of Yale University. Preliminary 327 experiments were tested to determine sample sizes, taking available recourses and ethical use into 328 account.

330 Psoriasis model

331 The psoriasis model used in this study is based on rIL-23 subcutaneous injections. The 500ng in $33220 \mu \mathrm{l}$ of rIL-23 (provided by Abbvie or purchased from R\&D Systems [scRNAseq experiments]) 333 was injected daily into the ear skin of anesthetized mice in 4 consecutive days. As a control $20 \mu 1$ 334 of PBS was used with the same injection intervals. For the second challenge experiment, we waited 
33510 days, monitoring the skin thickness before repeating 4-day injection regimen. Skin thickness

336 was measured daily with calipers. When indicated, FTY720 (1mg/kg) was dissolved in PBS and

337 administered i.p on day -1, 1 and 3 of the experiment. Skin tissue was collected on day 5 for

338 histology imaging, flow cytometry analysis or cell sorting.

340 Isolation of skin lymphocytes

341 Ventral and dorsal dermal sheets of ears were separated, minced and incubated in RPMI medium

342 containing $0.4 \mathrm{mg} \mathrm{ml}^{-1}$ Liberase TM (Roche Diagnostics) and 60ng/ul DNAseI (Sigma). After

343 digestion, the suspension was passed through and further mechanically disrupted with syringe

344 plunger and a 70uM cell strainer. Lymphocytes were enriched by gradient centrifugation in $27.5 \%$

345 Optiprep solution (Sigma) and RPMI medium containing 5\% Fetal Bovine Serum. Spleens were

346 mechanically disrupted using a syringe plunger in complete RPMI. Cells were filtered through 70-

$347 \mu \mathrm{m}$ nylon mesh and washed

$349 \quad$ Flow cytometry and cell sorting

350 Mouse ILCs were stained with monoclonal antibodies to CD45.2, CD90.2, lineage (CD4, CD8, 351 CD11b, CD11c, CD19, B220, NK1.1, Ter119, Gr1, FcEr1a), TCR $\beta$, TCR $\gamma$, CD3e. For 352 intracellular cytokine staining, cells were re-stimulated for $6 \mathrm{~h}$ at $37^{\circ} \mathrm{C}$ with phorbol 12353 myristate 13-acetate (PMA) (Sigma, $50 \mathrm{ng} \mathrm{ml}^{-1}$ ) and ionomycin (Sigma, $1 \mu \mathrm{g} \mathrm{ml} \mathrm{m}^{-1}$ ) in the 354 presence of Golgistop (BD Bioscience) added after initial 2h of stimulation. Next, cells were 355 fixed and stained with BS Cytofix/Cytoperm reagent (BD Biosciences) according to the 356 manufacturer's protocol. Intracellular cytokines were stained with antibodies to IL-13, IL-17A 
and IL-22. Total ILCs were sorted as live, CD45+, CD90+, lin- (CD4, CD8, CD11b, CD11c, CD19, B220, NK1.1, Ter119, Gr1, FcEr1a), CD3e- and TCR $\gamma / \delta$ - cells into PBS/0.2\%FBS.

\section{In-vitro ILC cultures}

361 For in vitro experiments, 5,000 ILCs were cultured per well of a 96-well round bottom plate in

362 Click's medium with $10 \mathrm{ng} \mathrm{m}^{-1} \mathrm{IL}-2$ (R\&D Systems) and $25 \mathrm{ng} \mathrm{ml}^{-1}$ IL-25 (R\&D Systems) with

$36310 \mathrm{ng} \mathrm{ml}^{-1}$, IL-33 (R\&D Systems) or IL-23 $25 \mathrm{ng} \mathrm{ml}^{-1}$ (Provided by Abbvie) with TGF $\beta 10 \mathrm{ng} \mathrm{ml}^{-}$

$364{ }^{1}$ (R\&D Systems) and IL-1 $\beta 10 \mathrm{ng} \mathrm{ml}^{-1}$ (R\&D Systems). Cells were collected for RNA extraction 365 and qRT-PCR after 5 days of culture in $37^{\circ} \mathrm{C}$ and $5 \% \mathrm{CO}_{2}$.

367 Adoptive ILC transfer

368 Total skin ILCs were FACS purified and collected to PBS 5\% serum. Cells were washed twice 369 with 1x PBS and injected (10,000 cells per mouse in 100ul) into retro-orbital vein of anesthetized $370 \mathrm{Rag}^{-/-} \mathrm{IL} 2 \mathrm{rg}^{-/-}$mice. IL-23 injection experiments were performed 14 days after the transfer.

\section{RNA extraction and Quantitative Real time PCR (qRT-PCR)}

373 RNA from in vitro cultures was isolated with RNeasy Mini Kit (QIAGEN) and qPCR was 374 performed using KAPA Probe Fast qPCR Master Mix 2x Kit (Kapa Biosystems, Wilmington, MA) 375 with TaqMan probes (Applied Biosystems) in a StepOne cycler (Applied Biosystems, Carlsbad, $376 \mathrm{CA})$. The $\mathrm{CT}$ values from duplicate qPCR reactions were extracted from the StepOne cycler 377 (Applied Biosystems, Carlsbad, CA) onto Excel spreadsheets and were analysed with the relative 378 quantification method $2^{\Delta \Delta \mathrm{CT}}$. 


\section{ATAC-seq}

381 Total ILCs sorted from naïve wild type mice were processed for ATAC-seq analysis according to 382 previously published protocol (Buenrostro, Giresi et al. 2013) with the low cell number input 383 version ( 5,000 ILCs). Libraries from two independent experiments were sequenced on 384 HiSeq2500 with 75bp paired end reads. Each sample was sequenced to a depth of 150 million 385 reads.

\section{$387 \quad$ ATAC-Seq data analysis}

388 Adapter sequences were trimmed using FASTX-Toolkit (version 0.0.13, 389 http://hannonlab.cshl.edu/fastx toolkit/), after which Bowtie2 (Langmead and Salzberg 2012) 390 was used to align the reads to the mm10 genome. Picard tools (version 2.9.0, 391 https://broadinstitute.github.io/picard/ ) were used to remove PCR duplicates. Bedtools was used 392 to convert the bam file to a bed file, and all mapped reads were offset by +4 bp for the positive 393 strand and $-5 \mathrm{bp}$ for the negative strand. Peaks were called for each sample using macs2 (Zhang, 394 Liu et al. 2008) using parameters --nomodel --nolambda --shiftsize 75. ATAC-seq peaks were 395 visualizaed with the Integrative Genomics Viewer (Robinson, Thorvaldsdottir et al. 2011, 396 Thorvaldsdottir, Robinson et al. 2013) along with publicly available ChIP-seq via Cistrome DB 397 (Liu, Ortiz et al. 2011).

\section{Single cell RNA-Seq}

400 Sorted cells were washed with PBS/0.04\% BSA and processed for droplet-based 3' end massively 401 parallel scRNA-seq: sorted ILCs were encapsulated into droplets, and libraries were prepared 
402 using Chromium Single Cell 3' Reagent Kits v2 according to the manufacturer's protocol (10X

403 Genomics). scRNA-seq libraries were sequenced using a 75 cycle Nextseq 500 high output V2 kit.

404

405 Single cell RNA-Seq data analysis

406 Initial data processing and QC. Gene counts were obtained by aligning reads to the mm 10

407 genome using CellRanger software (v1.3) (10x Genomics).

408

409 To remove doublets and poor-quality cells, cells were excluded from subsequent analysis if they 410 were outliers in their sample of origin in terms of number of genes or number of unique molecular 411 identifiers (UMIs), which eliminated 5.8-7.9\% of cells per sample (Fig. S4B), or outliers across 412 all samples in percentage of mitochondrial genes, which eliminated at most $0.5 \%$ of remaining

413 cells (Fig. S4C). Sample-specific cut-offs ranged from 575-2,400 genes per cell for the $\operatorname{Ragl}^{-/-}$ 414 untreated sample to 850-3,100 genes per cell for the WT induced sample.

416 Normalization. To normalize gene counts, we used a scaling factor that reflected the expected 417 number of UMIs in each sample (Fig. S4D), rather than scaling all cells to a constant size, as in 418 TPM (Wallrapp, Riesenfeld et al. 2017) Let $w_{s}$ be the mean number of UMIs per cell in sample $s$. 419 UMI counts for cells in sample $s$ were scaled to:

$42010,000 \times\left(w_{s} / w_{\mathrm{WT}}\right.$ naive $)$

421 Taking the log of scaled UMI counts gives the normalized expression values referred to as $\log$ TPX.

423 Determination of variable genes. We fit the count data to a null model based on a negative 424 binomial distribution that explains the expected technical variation for each gene, given its 
425 expression level, as previously described(Pandey, Shekhar et al. 2018). A gene was considered to 426 exhibit non-technical variability if it had mean counts above 0.005 and a coefficient of variation 427 at least $\log (0.5)$ times that predicted by the null model (Fig. S4E). We performed variable gene 428 selection separately for each sample as well as for pooled samples from WT mice and, separately, 429 from $\operatorname{Rag} 1^{-/}$mice. To reduce downstream technical effects of the variation in extremely highly 430 expressing genes, we then removed any genes that had mean counts above 4 in WT or, separately, 431 Rag $1^{-/-}$cells (these were mostly ribosomal protein genes). The resulting conservative set of 271 432 genes was then used for the singular value decomposition (SVD). We chose this approach to ensure 433 that noisy variable gene selection was not a cause of the heterogeneity in the "cloud". Note that 434 downstream results were qualitatively similar and robust to several parameter settings, which yield 435 variable gene sets of very different sizes, as well as to other selection approaches (including the 436 FindVariableGenes() function in Seurat) (Butler, Hoffman et al. 2018).

438 Dimensionality reduction, clustering, and visualization. We computed an SVD on $z$-scored

439 variable genes, as determined above, using Seurat's RunPCA() function, with the "weight.by.var" 440 parameter set to FALSE (Butler, Hoffman et al. 2018). Assessing the decrease in marginal 441 proportion of variance explained with larger components, we selected the top 18 eigenvectors for 442 subsequent analysis, and confirmed that the resulting analyses were not sensitive to this exact 443 choice. We used these components with Seurat's FindClusters() and RunTSNE() functions, with 444 other parameter settings set to default, to cluster the cells, and to separately create a $t$-stochastic 445 neighborhood embedding (tSNE) for visualization, respectively. As previously described, 446 FindClusters() optimizes a modularity function on a $k$-nearest-neighbor graph computed from the 447 top eigenvectors. 
449 Removal of non-ILC clusters. Based on expression of marker genes across clusters, we

450 determined that a few very distinct clusters were unlikely to be ILCs: cells in those clusters had

451 little expression of Ptprc (CD45), and high expression of Colla2, or Tie1 and Pecam1, or Krt15.

452 Cells from these non-ILC clusters were removed, and the steps of normalizing the data, selecting

453 variable genes, performing PCA, and creating a tSNE were repeated as before, but the top 20

454 components of the SVD were used for subsequent analysis. After these steps, 18,852 cell profiles

455 remained, with 4,619-4,857 cells per sample.

456

457 Topic modeling. We fit an LDA topic model on the full, sparse counts matrix (18,852 cells and 458 27,998 genes) using the FitGoM() function from the CountClust R package (Dey, Hsiao et al. 459 2017), with the number of clusters $K$ set to 15 and the "tol" tolera nce parameter set to 10 . This 460 package is heavily based on the maptpx $\mathrm{R}$ package, which implements a posterior maximization 461 approach to fitting the model (Taddy 2012). Some approaches to selecting an appropriate value of

$462 K$ rely on having labeled training data for the model. Since we do not have such a model, we fit 463 the model for a range of values and computed the Akaike and Bayesian information criteria (AIC 464 and BIC) using the estimated likelihood returned by FitGoM() (Fig. S2A). Since AIC and BIC risk 465 under- and over-penalizing the fit, respectively, we selected a value of $K$ at a point where the AIC 466 curve had begun to decrease less steeply and the BIC curve had begun to climb.

468 Diffusion maps. To select cells and genes for the construction of diffusion maps, a cell was 469 considered "highly weighted" for a topic if its weight for the topic was above a topic-specific 
471 to the exact choice of threshold. Cells were used in the large diffusion map (Fig. 3A) if they were

472 highly weighted for any of topics $2,4,8,11,13$, or 15, but not 6 or 7 (Fig. S3B and C). A gene

473 was considered to be in the "top $n$ genes" for a topic if it was returned by the CountClust function

474 ExtractTopFeatures(), which selects genes that are most critical for separating one topic from the

475 others (similar to differential expression analysis between clusters), with the following parameter

476 settings: top_features $=n$, method="poisson", options="min", shared=TRUE. For visualization, the

477 "Score" shown for top genes (Fig. 2C, Fig. S2D) was computed as $100^{*} x$, where $x$ is the Kullback-

478 Leibler divergence score output by ExtractTopFeatures(), and then plotted on a logarithmic scale.

479 Genes were included in the large diffusion map if they were in the top 50 genes for topics $2,4,8$,

48011,13 , or 15 , but not in the top 5 genes for any other topics. For the smaller diffusion map (Fig.

481 3C), cells and genes were selected in an analogous way, but only for the three topics 8,13 , and 15.

482 Overall, the larger diffusion map was computed on 7,888 cells and 245 genes, and the smaller one

483 on 3,785 cells and 130 genes. To build the diffusion map, we gave the expression data for these

484 cells and genes as input to the DiffusionMap() function from the destiny R package (Angerer,

485 Haghverdi et al. 2016), with parameter settings $k=50$ and sigma="local".

\section{Acknowledgments}

488 We would like to thank Ania Hupalowska and Leslie Gaffney for help in manuscript preparation.

489 This work was supported in part by grants (YAP-013-2015) provided by AbbVie (RAF, PB). Work

490 supported by the Klarman Cell Observatory (AR) and HHMI (AR and RAF). AR is an SAB

491 member of ThermoFisher Scientific, Syros Pharmaceuticals, and Driver Group and a founder of

492 Celsius Therapeutics. 


\section{References}

495

Ahn, J., Y. Yuan, G. Parmigiani, M. B. Suraokar, L. Diao, Wistuba, II and W. Wang (2013). "DeMix: deconvolution for mixed cancer transcriptomes using raw measured data." Bioinformatics 29(15): 1865-1871.

Angerer, P., L. Haghverdi, M. Buttner, F. J. Theis, C. Marr and F. Buettner (2016). "destiny: diffusion maps for large-scale single-cell data in R." Bioinformatics 32(8): 1241-1243. Bal, S. M., J. H. Bernink, M. Nagasawa, J. Groot, M. M. Shikhagaie, K. Golebski, C. M. van Drunen, R. Lutter, R. E. Jonkers, P. Hombrink, M. Bruchard, J. Villaudy, J. M. Munneke, W. Fokkens, J. S. Erjefalt, H. Spits and X. R. Ros (2016). "IL-1beta, IL-4 and IL-12 control the fate of group 2 innate lymphoid cells in human airway inflammation in the lungs." Nat Immunol 17(6): 636-645.

Bernink, J. H., L. Krabbendam, K. Germar, E. de Jong, K. Gronke, M. Kofoed-Nielsen, J. M. Munneke, M. D. Hazenberg, J. Villaudy, C. J. Buskens, W. A. Bemelman, A. Diefenbach, B. Blom and H. Spits (2015). "Interleukin-12 and -23 Control Plasticity of CD127(+) Group 1 and Group 3 Innate Lymphoid Cells in the Intestinal Lamina Propria." Immunity 43(1): 146-160.

Blei, D. M., Ng, A.Y., Jordan, M.I. (2003). "Latent Dirichlet Allocation." Journal of Machine Learning Research 3: 29.

Buenrostro, J. D., P. G. Giresi, L. C. Zaba, H. Y. Chang and W. J. Greenleaf (2013). "Transposition of native chromatin for fast and sensitive epigenomic profiling of open chromatin, DNA-binding proteins and nucleosome position." Nat Methods 10(12): 1213-1218.

Butler, A., P. Hoffman, P. Smibert, E. Papalexi and R. Satija (2018). "Integrating single-cell transcriptomic data across different conditions, technologies, and species." Nat Biotechnol 36(5): 411-420.

Cai, Y., X. Shen, C. Ding, C. Qi, K. Li, X. Li, V. R. Jala, H. G. Zhang, T. Wang, J. Zheng and J. Yan (2011). "Pivotal role of dermal IL-17-producing gammadelta T cells in skin inflammation." Immunity 35(4): 596-610.

Cao, Z., X. Sun, B. Icli, A. K. Wara and M. W. Feinberg (2010). "Role of Kruppel-like factors in leukocyte development, function, and disease." Blood 116(22): 4404-4414.

Carlson, C. M., B. T. Endrizzi, J. Wu, X. Ding, M. A. Weinreich, E. R. Walsh, M. A. Wani, J. B. Lingrel, K. A. Hogquist and S. C. Jameson (2006). "Kruppel-like factor 2 regulates thymocyte and T-cell migration." Nature 442(7100): 299-302.

Carrelha, J., Y. Meng, L. M. Kettyle, T. C. Luis, R. Norfo, V. Alcolea, H. Boukarabila, F. Grasso, A. Gambardella, A. Grover, K. Hogstrand, A. M. Lord, A. Sanjuan-Pla, P. S. Woll, C. Nerlov and S. E. W. Jacobsen (2018). "Hierarchically related lineage-restricted fates of multipotent haematopoietic stem cells." Nature 554(7690): 106-111.

Cella, M., K. Otero and M. Colonna (2010). "Expansion of human NK-22 cells with IL-7, IL-2, and IL-1beta reveals intrinsic functional plasticity." Proc Natl Acad Sci U S A 107(24): 10961-10966. Ciofani, M., A. Madar, C. Galan, M. Sellars, K. Mace, F. Pauli, A. Agarwal, W. Huang, C. N. Parkhurst, M. Muratet, K. M. Newberry, S. Meadows, A. Greenfield, Y. Yang, P. Jain, F. K. Kirigin, C. Birchmeier, E. F. Wagner, K. M. Murphy, R. M. Myers, R. Bonneau and D. R. Littman (2012). "A validated regulatory network for Th17 cell specification." Cell 151(2): 289-303. Dey, K. K., C. J. Hsiao and M. Stephens (2017). "Visualizing the structure of RNA-seq expression data using grade of membership models." PLoS Genet 13(3): e1006599. 
Dyring-Andersen, B., C. Geisler, C. Agerbeck, J. P. Lauritsen, S. D. Gudjonsdottir, L. Skov and C. M. Bonefeld (2014). "Increased number and frequency of group 3 innate lymphoid cells in nonlesional psoriatic skin." Br J Dermatol 170(3): 609-616. Esplugues, E., S. Huber, N. Gagliani, A. E. Hauser, T. Town, Y. Y. Wan, W. O'Connor, Jr., A. Rongvaux, N. Van Rooijen, A. M. Haberman, Y. Iwakura, V. K. Kuchroo, J. K. Kolls, J. A. Bluestone, K. C. Herold and R. A. Flavell (2011). "Control of TH17 cells occurs in the small intestine." Nature 475(7357): 514-518. Galloway, A., A. Saveliev, S. Lukasiak, D. J. Hodson, D. Bolland, K. Balmanno, H. Ahlfors, E. Monzon-Casanova, S. C. Mannurita, L. S. Bell, S. Andrews, M. D. Diaz-Munoz, S. J. Cook, A. Corcoran and M. Turner (2016). "RNA-binding proteins ZFP36L1 and ZFP36L2 promote cell quiescence." Science 352(6284): 453-459.

548 Gasteiger, G., X. Fan, S. Dikiy, S. Y. Lee and A. Y. Rudensky (2015). "Tissue residency of innate lymphoid cells in lymphoid and nonlymphoid organs." Science 350(6263): 981-985. Gury-BenAri, M., C. A. Thaiss, N. Serafini, D. R. Winter, A. Giladi, D. Lara-Astiaso, M. Levy, T. M. Salame, A. Weiner, E. David, H. Shapiro, M. Dori-Bachash, M. Pevsner-Fischer, E. Lorenzo-Vivas, H. Keren-Shaul, F. Paul, A. Harmelin, G. Eberl, S. Itzkovitz, A. Tanay, J. P. Di Santo, E. Elinav and I. Amit (2016). "The Spectrum and Regulatory Landscape of Intestinal Innate Lymphoid Cells Are Shaped by the Microbiome." Cell 166(5): 1231-1246 e1213. Haghverdi, L., M. Buttner, F. A. Wolf, F. Buettner and F. J. Theis (2016). "Diffusion pseudotime robustly reconstructs lineage branching." Nat Methods 13(10): 845-848. Huang, Y., L. Guo, J. Qiu, X. Chen, J. Hu-Li, U. Siebenlist, P. R. Williamson, J. F. Urban, Jr. and W. E. Paul (2015). "IL-25-responsive, lineage-negative KLRG1(hi) cells are multipotential 'inflammatory' type 2 innate lymphoid cells." Nat Immunol 16(2): 161-169. Huang, Y., K. Mao, X. Chen, M. A. Sun, T. Kawabe, W. Li, N. Usher, J. Zhu, J. F. Urban, Jr., W. E. Paul and R. N. Germain (2018). "S1P-dependent interorgan trafficking of group 2 innate lymphoid cells supports host defense." Science 359(6371): 114-119. Kumari, S., S. Curado, V. Mayya and M. L. Dustin (2014). "T cell antigen receptor activation and actin cytoskeleton remodeling." Biochim Biophys Acta 1838(2): 546-556. Kuwano, Y., C. M. Prazma, N. Yazawa, R. Watanabe, N. Ishiura, A. Kumanogoh, H. Okochi, K. Tamaki, M. Fujimoto and T. F. Tedder (2007). "CD83 influences cell-surface MHC class II expression on B cells and other antigen-presenting cells." Int Immunol 19(8): 977-992. Langmead, B. and S. L. Salzberg (2012). "Fast gapped-read alignment with Bowtie 2." Nat Methods 9(4): 357-359. Laurenti, E. and B. Gottgens (2018). "From haematopoietic stem cells to complex differentiation landscapes." Nature 553(7689): 418-426.

Li, P., R. Spolski, W. Liao, L. Wang, T. L. Murphy, K. M. Murphy and W. J. Leonard (2012). "BATFJUN is critical for IRF4-mediated transcription in T cells." Nature 490(7421): 543-546.

Li, Z., T. Hodgkinson, E. J. Gothard, S. Boroumand, R. Lamb, I. Cummins, P. Narang, A. Sawtell, J. Coles, G. Leonov, A. Reboldi, C. D. Buckley, T. Cupedo, C. Siebel, A. Bayat, M. C. Coles and C. A. Ambler (2016). "Epidermal Notch1 recruits RORgamma(+) group 3 innate lymphoid cells to orchestrate normal skin repair." Nat Commun 7: 11394.

580 Lim, A. I., S. Menegatti, J. Bustamante, L. Le Bourhis, M. Allez, L. Rogge, J. L. Casanova, H. Yssel and J. P. Di Santo (2016). "IL-12 drives functional plasticity of human group 2 innate lymphoid cells." J Exp Med 213(4): 569-583. 
581

582

583

584

585

586

587

588

589

590

591

592

593

594

595

596

597

598

599

600

601

602

603

604

605

606

607

608

609

610

611

612

613

614

615

616

617

618

619

620

621

622

623

Lindsay, J., Mandoiu, I., Nelson, C. (2013). "Gene Expression Deconvolution using Single-cells." Proceedings of the 2013 American Association of Human Genetics meeting.

Liu, T., J. A. Ortiz, L. Taing, C. A. Meyer, B. Lee, Y. Zhang, H. Shin, S. S. Wong, J. Ma, Y. Lei, U. J. Pape, M. Poidinger, Y. Chen, K. Yeung, M. Brown, Y. Turpaz and X. S. Liu (2011). "Cistrome: an integrative platform for transcriptional regulation studies." Genome Biol 12(8): R83.

Matloubian, M., C. G. Lo, G. Cinamon, M. J. Lesneski, Y. Xu, V. Brinkmann, M. L. Allende, R. L. Proia and J. G. Cyster (2004). "Lymphocyte egress from thymus and peripheral lymphoid organs is dependent on S1P receptor 1." Nature 427(6972): 355-360.

Nograles, K. E., L. C. Zaba, E. Guttman-Yassky, J. Fuentes-Duculan, M. Suarez-Farinas, I. Cardinale, A. Khatcherian, J. Gonzalez, K. C. Pierson, T. R. White, C. Pensabene, I. Coats, I. Novitskaya, M. A. Lowes and J. G. Krueger (2008). "Th17 cytokines interleukin (IL)-17 and IL-22 modulate distinct inflammatory and keratinocyte-response pathways." Br J Dermatol 159(5): 1092-1102.

Nussbaum, J. C., S. J. Van Dyken, J. von Moltke, L. E. Cheng, A. Mohapatra, A. B. Molofsky, E. E. Thornton, M. F. Krummel, A. Chawla, H. E. Liang and R. M. Locksley (2013). "Type 2 innate lymphoid cells control eosinophil homeostasis." Nature 502(7470): 245-248.

Ohne, Y., J. S. Silver, L. Thompson-Snipes, M. A. Collet, J. P. Blanck, B. L. Cantarel, A. M. Copenhaver, A. A. Humbles and Y. J. Liu (2016). "IL-1 is a critical regulator of group 2 innate lymphoid cell function and plasticity." Nat Immunol 17(6): 646-655.

Pandey, S., K. Shekhar, A. Regev and A. F. Schier (2018). "Comprehensive Identification and Spatial Mapping of Habenular Neuronal Types Using Single-Cell RNA-Seq." Curr Biol 28(7): 10521065 e1057.

Pantelyushin, S., S. Haak, B. Ingold, P. Kulig, F. L. Heppner, A. A. Navarini and B. Becher (2012).

"Rorgammat+ innate lymphocytes and gammadelta T cells initiate psoriasiform plaque formation in mice." J Clin Invest 122(6): 2252-2256.

Pritchard, J. K., M. Stephens and P. Donnelly (2000). "Inference of population structure using multilocus genotype data." Genetics 155(2): 945-959.

Quon, G., S. Haider, A. G. Deshwar, A. Cui, P. C. Boutros and Q. Morris (2013). "Computational purification of individual tumor gene expression profiles leads to significant improvements in prognostic prediction." Genome Med 5(3): 29.

Repsilber, D., S. Kern, A. Telaar, G. Walzl, G. F. Black, J. Selbig, S. K. Parida, S. H. Kaufmann and M. Jacobsen (2010). "Biomarker discovery in heterogeneous tissue samples -taking the in-silico deconfounding approach." BMC Bioinformatics 11: 27.

Ricardo-Gonzalez, R. R., S. J. Van Dyken, C. Schneider, J. Lee, J. C. Nussbaum, H. E. Liang, D. Vaka, W. L. Eckalbar, A. B. Molofsky, D. J. Erle and R. M. Locksley (2018). "Tissue signals imprint ILC2 identity with anticipatory function." Nat Immunol.

Robinette, M. L., A. Fuchs, V. S. Cortez, J. S. Lee, Y. Wang, S. K. Durum, S. Gilfillan, M. Colonna and C. Immunological Genome (2015). "Transcriptional programs define molecular characteristics of innate lymphoid cell classes and subsets." Nat Immunol 16(3): 306-317. Robinson, J. T., H. Thorvaldsdottir, W. Winckler, M. Guttman, E. S. Lander, G. Getz and J. P. Mesirov (2011). "Integrative genomics viewer." Nat Biotechnol 29(1): 24-26.

Roediger, B., R. Kyle, K. H. Yip, N. Sumaria, T. V. Guy, B. S. Kim, A. J. Mitchell, S. S. Tay, R. Jain, E. Forbes-Blom, X. Chen, P. L. Tong, H. A. Bolton, D. Artis, W. E. Paul, B. Fazekas de St Groth, M. A. 
624 Grimbaldeston, G. Le Gros and W. Weninger (2013). "Cutaneous immunosurveillance and regulation of inflammation by group 2 innate lymphoid cells." Nat Immunol 14(6): 564-573. Salerno, F., S. Engels, M. van den Biggelaar, F. P. J. van Alphen, A. Guislain, W. Zhao, D. L. Hodge, S. E. Bell, J. P. Medema, M. von Lindern, M. Turner, H. A. Young and M. C. Wolkers (2018). "Translational repression of pre-formed cytokine-encoding mRNA prevents chronic activation of memory T cells." Nat Immunol. Schroder, B. (2016). "The multifaceted roles of the invariant chain CD74--More than just a chaperone." Biochim Biophys Acta 1863(6 Pt A): 1269-1281. Schwartz, R. and S. E. Shackney (2010). "Applying unmixing to gene expression data for tumor phylogeny inference." BMC Bioinformatics 11: 42. Shen-Orr, S. S., R. Tibshirani, P. Khatri, D. L. Bodian, F. Staedtler, N. M. Perry, T. Hastie, M. M. Sarwal, M. M. Davis and A. J. Butte (2010). "Cell type-specific gene expression differences in complex tissues." Nat Methods 7(4): 287-289. Silver, J. S., J. Kearley, A. M. Copenhaver, C. Sanden, M. Mori, L. Yu, G. H. Pritchard, A. A. Berlin, C. A. Hunter, R. Bowler, J. S. Erjefalt, R. Kolbeck and A. A. Humbles (2016). "Inflammatory triggers associated with exacerbations of COPD orchestrate plasticity of group 2 innate lymphoid cells in the lungs." Nat Immunol 17(6): 626-635. Spencer, S. P., C. Wilhelm, Q. Yang, J. A. Hall, N. Bouladoux, A. Boyd, T. B. Nutman, J. F. Urban, Jr., J. Wang, T. R. Ramalingam, A. Bhandoola, T. A. Wynn and Y. Belkaid (2014). "Adaptation of innate lymphoid cells to a micronutrient deficiency promotes type 2 barrier immunity." Science 343(6169): 432-437. Sutton, V. R., A. J. Brennan, S. Ellis, J. Danne, K. Thia, M. R. Jenkins, I. Voskoboinik, G. Pejler, R. W. Johnstone, D. M. Andrews and J. A. Trapani (2016). "Serglycin determines secretory granule repertoire and regulates natural killer cell and cytotoxic T lymphocyte cytotoxicity." FEBS J 283(5): 947-961.

Taddy, M. (2012). "On Estimation and Selection for Topic Models." Proceedings of Machine Learning Research 22: 1184--1193.

Teunissen, M. B., J. M. Munneke, J. H. Bernink, P. I. Spuls, P. C. Res, A. Te Velde, S. Cheuk, M. W. Brouwer, S. P. Menting, L. Eidsmo, H. Spits, M. D. Hazenberg and J. Mjosberg (2014). "Composition of innate lymphoid cell subsets in the human skin: enrichment of NCR(+) ILC3 in lesional skin and blood of psoriasis patients." J Invest Dermatol 134(9): 2351-2360. Teunissen, M. B. M., J. M. Munneke, J. H. Bernink, P. I. Spuls, P. C. M. Res, A. Te Velde, S. Cheuk, M. W. D. Brouwer, S. P. Menting, L. Eidsmo, H. Spits, M. D. Hazenberg and J. Mjosberg (2014). "Composition of innate lymphoid cell subsets in the human skin: enrichment of NCR(+) ILC3 in lesional skin and blood of psoriasis patients." J Invest Dermatol 134(9): 2351-2360. Thorvaldsdottir, H., J. T. Robinson and J. P. Mesirov (2013). "Integrative Genomics Viewer (IGV): high-performance genomics data visualization and exploration." Brief Bioinform 14(2): 178-192. Trapnell, C., D. Cacchiarelli, J. Grimsby, P. Pokharel, S. Li, M. Morse, N. J. Lennon, K. J. Livak, T. S. Mikkelsen and J. L. Rinn (2014). "The dynamics and regulators of cell fate decisions are revealed by pseudotemporal ordering of single cells." Nat Biotechnol 32(4): 381-386.

666 Tsukada, J., Y. Yoshida, Y. Kominato and P. E. Auron (2011). "The CCAAT/enhancer (C/EBP) family of basic-leucine zipper (bZIP) transcription factors is a multifaceted highly-regulated system for gene regulation." Cytokine 54(1): 6-19. 
667 Tusi, B. K., S. L. Wolock, C. Weinreb, Y. Hwang, D. Hidalgo, R. Zilionis, A. Waisman, J. R. Huh, A. 668 M. Klein and M. Socolovsky (2018). "Population snapshots predict early haematopoietic and 669 erythroid hierarchies." Nature 555(7694): 54-60.

670 Villanova, F., B. Flutter, I. Tosi, K. Grys, H. Sreeneebus, G. K. Perera, A. Chapman, C. H. Smith, P. 671 Di Meglio and F. O. Nestle (2014). "Characterization of innate lymphoid cells in human skin and 672 blood demonstrates increase of NKp44+ ILC3 in psoriasis." J Invest Dermatol 134(4): 984-991. 673 Villanova, F., B. Flutter, I. Tosi, K. Grys, H. Sreeneebus, G. K. Perera, A. Chapman, C. H. Smith, P. 674 Di Meglio and F. O. Nestle (2014). "Characterization of innate lymphoid cells in human skin and 675 blood demonstrates increase of NKp44+ ILC3 in psoriasis." J Invest Dermatol 134(4): 984-991.

676 Wallrapp, A., S. J. Riesenfeld, P. R. Burkett, R. E. Abdulnour, J. Nyman, D. Dionne, M. Hofree, M. 677 S. Cuoco, C. Rodman, D. Farouq, B. J. Haas, T. L. Tickle, J. J. Trombetta, P. Baral, C. S. N. Klose, T. 678 Mahlakoiv, D. Artis, O. Rozenblatt-Rosen, I. M. Chiu, B. D. Levy, M. S. Kowalczyk, A. Regev and V. 679 K. Kuchroo (2017). "The neuropeptide NMU amplifies ILC2-driven allergic lung inflammation." 680 Nature 549(7672): 351-356.

681 Wang, N., T. Gong, R. Clarke, L. Chen, M. Shih le, Z. Zhang, D. A. Levine, J. Xuan and Y. Wang 682 (2015). "UNDO: a Bioconductor R package for unsupervised deconvolution of mixed gene 683 expressions in tumor samples." Bioinformatics 31(1): 137-139.

684 Yang, J., S. Hu, L. Zhao, D. H. Kaplan, G. H. Perdew and N. Xiong (2016). "Selective programming 685 of CCR10(+) innate lymphoid cells in skin-draining lymph nodes for cutaneous homeostatic 686 regulation." Nat Immunol 17(1): 48-56.

687 Yosef, N., A. K. Shalek, J. T. Gaublomme, H. Jin, Y. Lee, A. Awasthi, C. Wu, K. Karwacz, S. Xiao, M. 688 Jorgolli, D. Gennert, R. Satija, A. Shakya, D. Y. Lu, J. J. Trombetta, M. R. Pillai, P. J. Ratcliffe, M. L. 689 Coleman, M. Bix, D. Tantin, H. Park, V. K. Kuchroo and A. Regev (2013). "Dynamic regulatory 690 network controlling TH17 cell differentiation." Nature 496(7446): 461-468.

691 Zhang, K., X. Xu, M. A. Pasha, C. W. Siebel, A. Costello, A. Haczku, K. MacNamara, T. Liang, J.

692 Zhu, A. Bhandoola, I. Maillard and Q. Yang (2017). "Cutting Edge: Notch Signaling Promotes the 693 Plasticity of Group-2 Innate Lymphoid Cells." J Immunol 198(5): 1798-1803.

694 Zhang, Y., T. Liu, C. A. Meyer, J. Eeckhoute, D. S. Johnson, B. E. Bernstein, C. Nusbaum, R. M. 695 Myers, M. Brown, W. Li and X. S. Liu (2008). "Model-based analysis of ChIP-Seq (MACS)." 696 Genome Biol 9(9): R137.

697 Zhong, C., K. Cui, C. Wilhelm, G. Hu, K. Mao, Y. Belkaid, K. Zhao and J. Zhu (2016). "Group 3 698 innate lymphoid cells continuously require the transcription factor GATA-3 after commitment." 699 Nat Immunol 17(2): 169-178. 


\section{Figure 1. An epigenetically poised, heterogenous population of tissue-resident ILCs drive initial IL-23-induced}

pathology. (A) Study overview. From left: Psoriasis mouse model is based on a series of subcutaneous IL-23 injections in WT, $\operatorname{Ragl}^{-/-}$and $T c d r^{-/-}$mice, phenotypic measurement of skin thickness, and tissue collection and cell isolation for assessment by scRNA-seq, in vitro assays and cytokine expression. $(\mathbf{B}, \mathbf{C})$ Tissue resident ILCs are necessary and sufficient for increase in ear skin thickness in response to IL-23 treatment. Increase in skin thickness (mm, y axis) over time (days, $\mathrm{x}$ axis) in (B) WT (black), $R a g 1^{-/-}$(lack all T and B cells, blue), $\operatorname{Rag}^{-/-} I l 2 \mathrm{rg}^{-/-}$mice (also lack ILCs, magenta), and $\mathrm{TCR} \gamma \delta^{-/-}$( lack $\gamma \delta \mathrm{T}$ cells, grey) (n=7 for each group) as well as in (C) $R a g 2^{-/-} \mathrm{Il}_{2} \mathrm{rg}^{-/-}$mice with (blue) and without (black) intravenously transferred ILCs ( $\mathrm{n}=4$ for each group). (D) FTY720 blocks white blood cell circulation. Total circulatory white blood cell (WBC) numbers $\left(10^{3} / \mu 1\right.$; y axis) in untreated ("Non-Tx") and FTY720-treated ("FTY720Tx”), WT and $\mathrm{Rag}^{-/-}$mice. (E) IL23-dependent increases in ear skin thickness does not require circulating cells. Increase in skin thickness (mm, y axis) over time (days, x axis) following IL-23 treatment, in WT (top) and Rag $^{-/-}$(bottom) mice, with (red) and without (black) FTY720-treatment (Methods) (n=3 WT both groups, $\mathrm{n}=2$ Rag $1^{-/-}$NonTX n=4 Rag $1^{-/-}$ FTY720). (F,G) A secondary challenge with IL-23 increases susceptibility. Increase in skin thickness (mm, y axis) over time (days, $\mathrm{x}$ axis; top) or at the end (bottom) of a primary (white bar) or secondary (blue bars) challenge with either IL$23(\mathrm{n}=14)$ or saline control (PBS) $(\mathrm{n}=5)$. (H) ILCs in untreated mice are epigenetically poised to become ILC3s. Mapped ATAC-seq reads (top track) at the Il22 (left) and Il5 (right) promoter loci (bottom track) from sorted skin ILCs from untreated mice, show open chromatin peaks (beige and blue bars) at key TF binding sites (beige), previously identified in $\mathrm{CD}^{+} \mathrm{T}$ cell ChIP-seq data (middle tracks), and at the TSS of $I l 5$ but not $I l 22$ (blue). (I,J) ILC heterogeneity highlighted by scRNA-seq. $t$-Distributed stochastic neighbor embedding (tSNE) of 27,998 single cell (dots) profiles (Methods) colored by either in vivo treatment and genotype (i), or by cluster assignment or expression of key genes (color bar, $\log$ TPX (Methods)) (J). Annotated clusters (J, top left) include a $R a g 1^{-/-}$-specific cluster (A) expressing the ILC1associated gene $K l r d 1$, cycling cells (B), an $I l 22$-high cluster co-expressing $I l 13$ (C), and a heterogeneous "cloud" (D), without discrete boundaries between clusters yet with multiple patterns of graded gene expression. Error bars, SD; $* * \mathrm{p}<0.021, * * * \mathrm{p}<0.0002, * * * * \mathrm{p}<0.0001$ by unpaired t test (b) or two-way ANOVA (d, e, g). 
bioRxiv preprint doi: https://doi.org/10.1101/461228; this version posted November 12, 2018. The copyright holder for this preprint (which was not certified by peer review) is the author/funder. All rights reserved. No reuse allowed without permission.

Figure 1

(1) Mouse model

(2) Subcutaneous ear injection

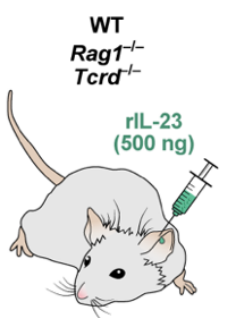

procedure:

\begin{tabular}{|c|c|c|}
\hline $\begin{array}{l}\text { Skin thickness } \\
\text { measurement }\end{array}$ & Day & $\begin{array}{c}\begin{array}{c}\text { rllL-23 } \\
\text { injection }\end{array} \\
\end{array}$ \\
\hline |"'"'"'"| & 0 & 一필 \\
\hline |"'"'"'|" & 1 & -1 \\
\hline |"'"'"'"'| & 2 & - \\
\hline |"'"'"'"'|" & 3 & -1 \\
\hline |"'"'"'|" & 4 & $\begin{array}{l}\text { Sample } \\
\text { collection }\end{array}$ \\
\hline
\end{tabular}

(3) Tissue collection and processing

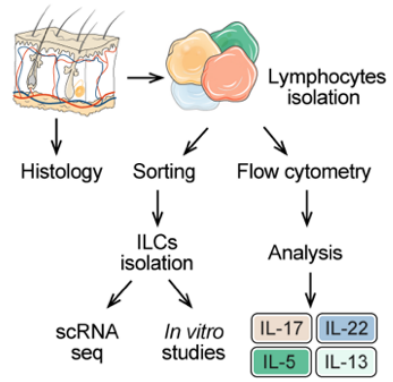

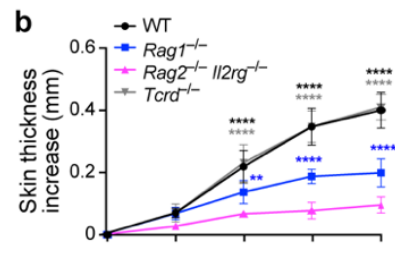

c

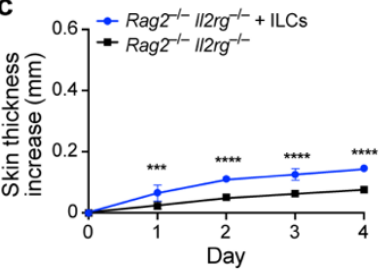

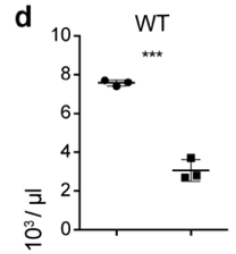

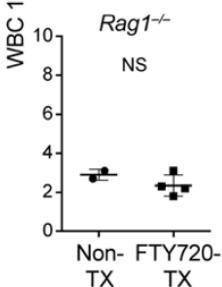

e

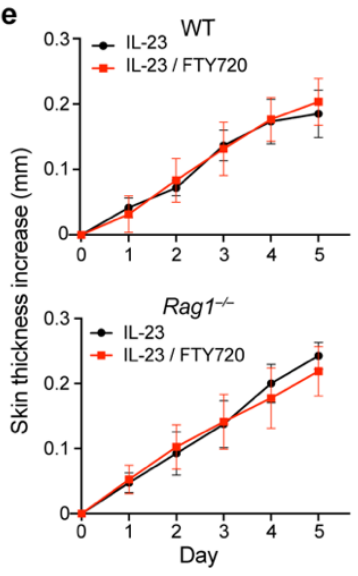

i

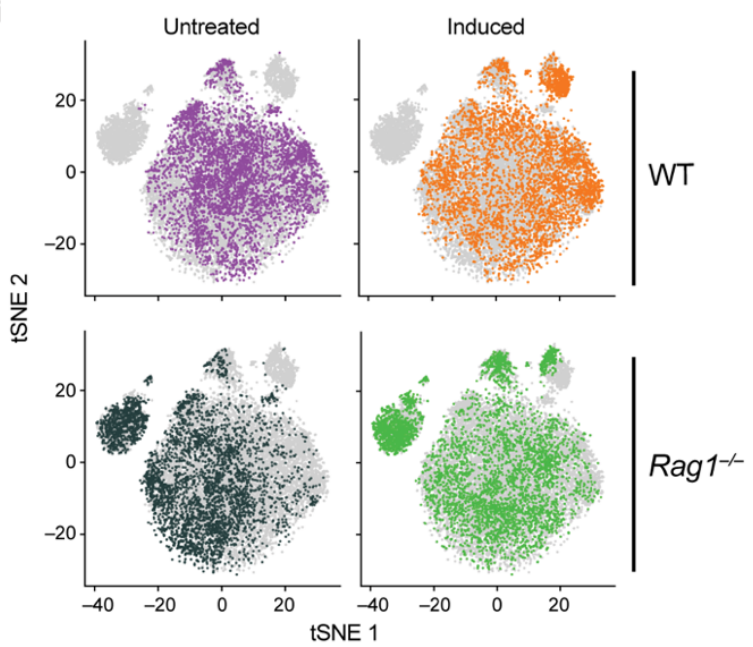

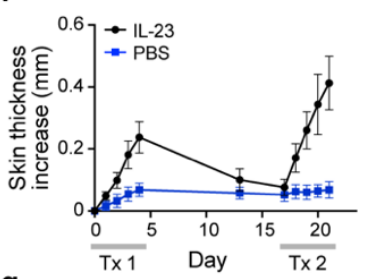
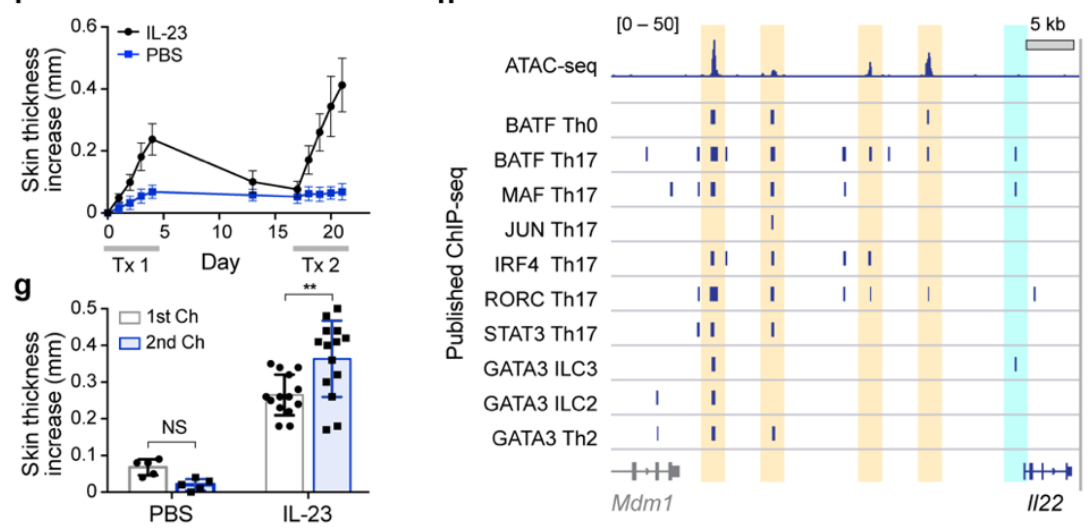

j

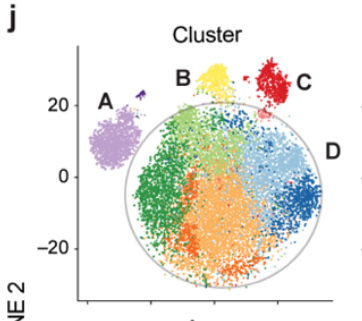

$\underset{\mathrm{W}}{\mathrm{W}}$

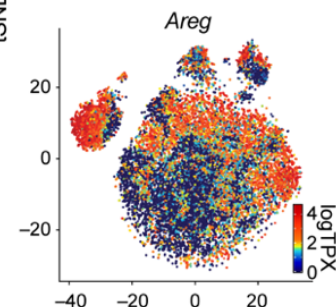

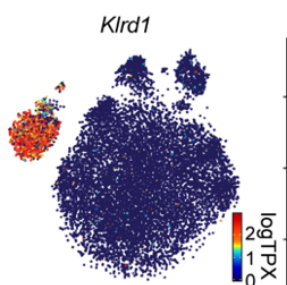
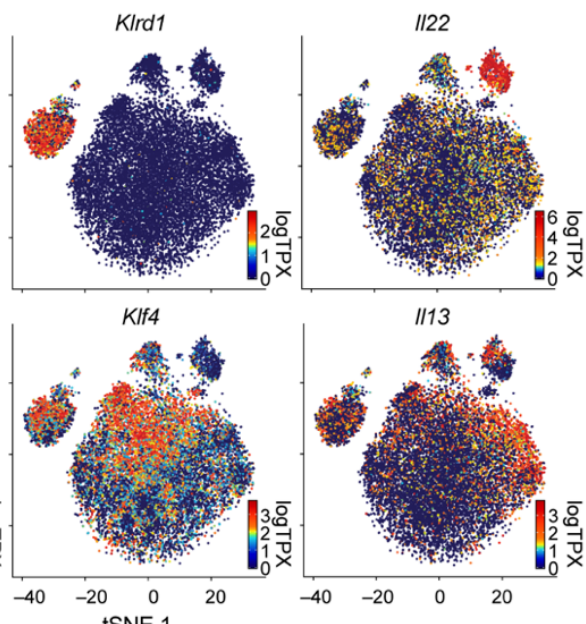
Figure 2. Topic modeling of skin ILCs highlights repressive, quiescent-like state and multiple, distinct states of

activation combined in cells. (A) Topic model concept in the context of single cell expression. Topics (top) consist of genes (middle), with distinct weights (gradient, Methods) based on their importance in the topic. Cells (bottom), are scored based on the contribution of each topic (color) in them; a cell can thus have multiple topics. (B-E), Results of LDA on ILCs with 15 topics (Methods). (B,C) Topics reveal complex relationships among clusters. TSNE of cells colored if they are highly weighted for Topic 11 and gray otherwise, with color code reflecting cluster membership as in Fig. 1J (B, left), or by expression (color bar, $\log$ TPX (Methods)) of Cd83, a Topic-11 associated gene (b, right). Analogous plots for Topic 13 (c, left) and its associated gene $\operatorname{Srgn}(\mathbf{C}$, right). (D,E) Topics with high weights in cells from untreated (Topics 2, 8, and 11) vs. induced (Topics 4, 13, and 15) conditions. (D) For each topic shown are a bar plot of top scoring genes (y axis), ranked by a score (x axis, logarithmic scale) of how well the gene distinguishes this from other topics (Methods); a tSNE (as in Fig. 1I) with cells colored by the topic's weight in the cells (column $j$ of the cell-by-topic weight matrix $\theta\left(\theta_{*}\right)$ for Topic $\left.j\right)$; and a graph of the empirical cumulative density function (y axis) of topic weights $\theta_{*, j}$ (x axis) for cells grouped by treatment or genotype (as in Fig. 1H). (E) Examples of topic-associated genes. Gene expression (y axis, $\log$ TPX) as a smoothed function of the topic weight $\left(\log \theta_{*_{j}}, \mathrm{x}\right.$ axis), for each of the topics highlighted in $\mathbf{D}$ (color) 
bioRxiv preprint doi: https://doi.org/10.1101/461228; this version posted November 12, 2018. The copyright holder for this preprint (which was not certified by peer review) is the author/funder. All rights reserved. No reuse allowed without permission.

\section{Figure 2}

a

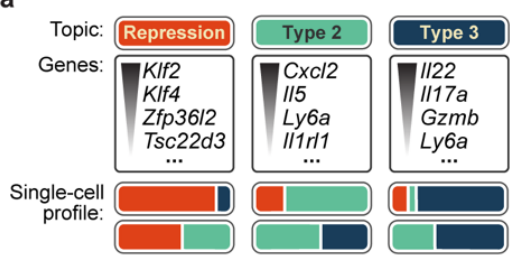

d
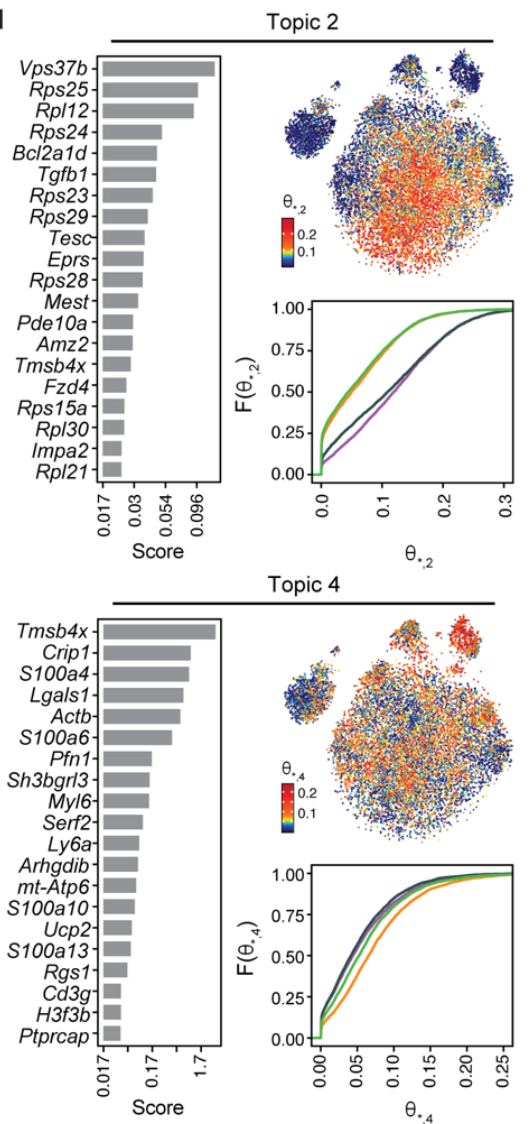

Topic 4

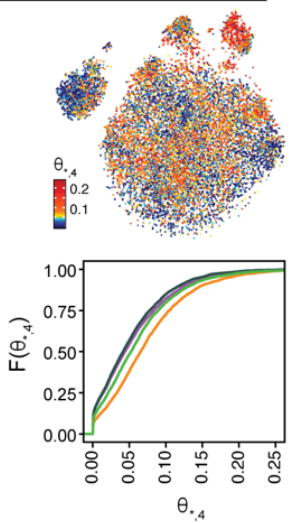

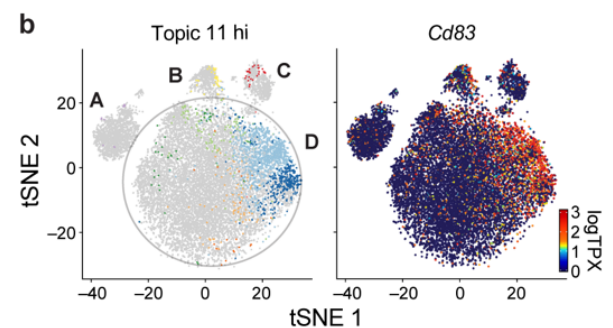
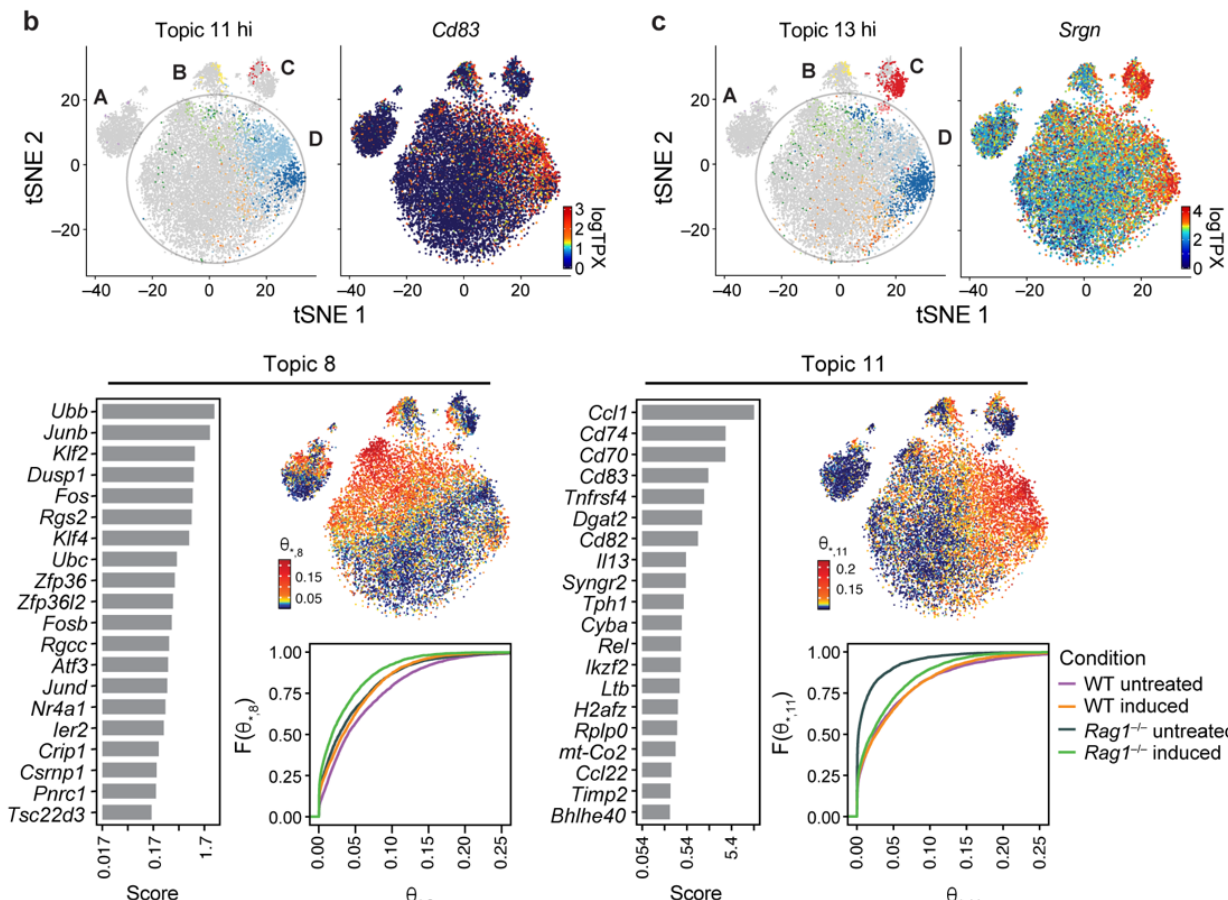

Topic 8

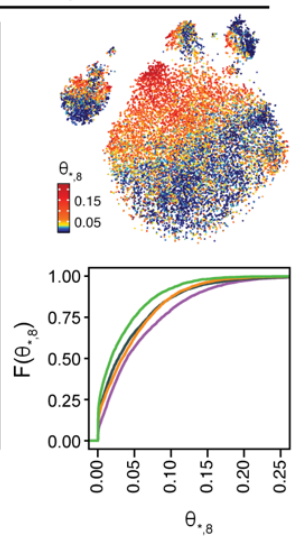

Topic 13
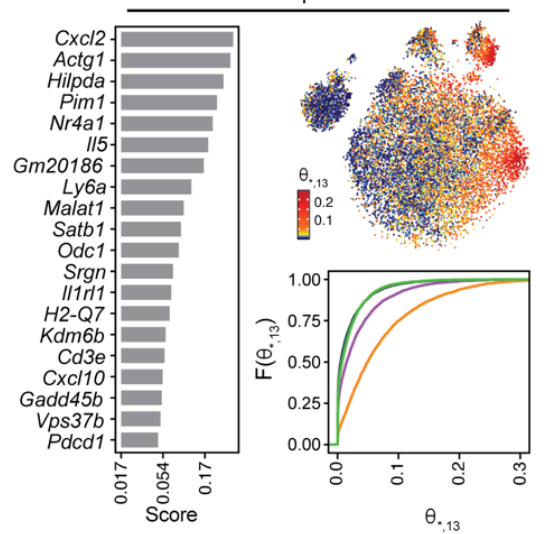

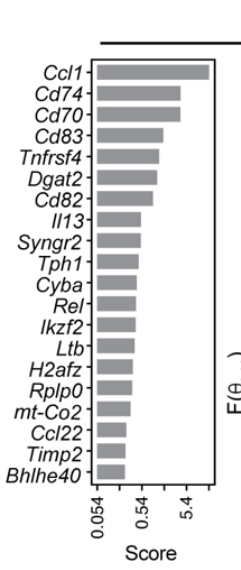

Topic 11
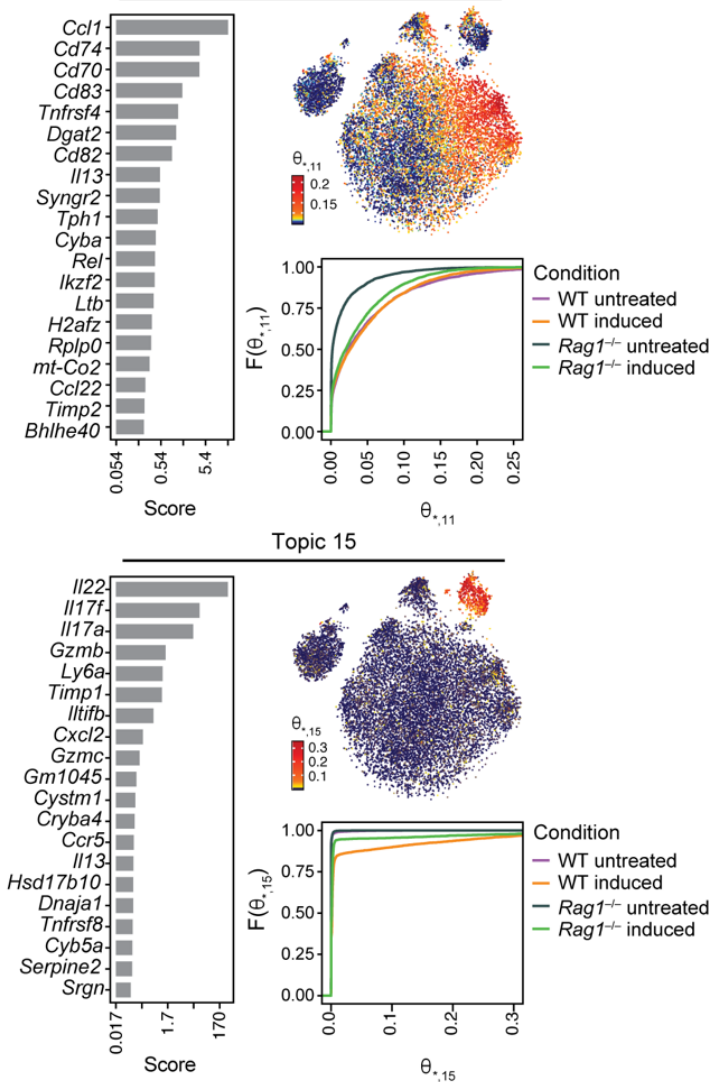
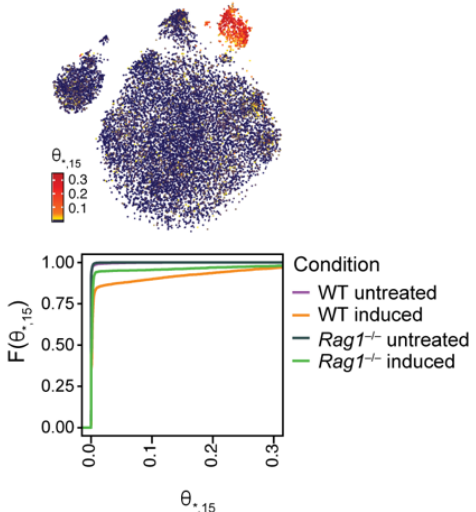
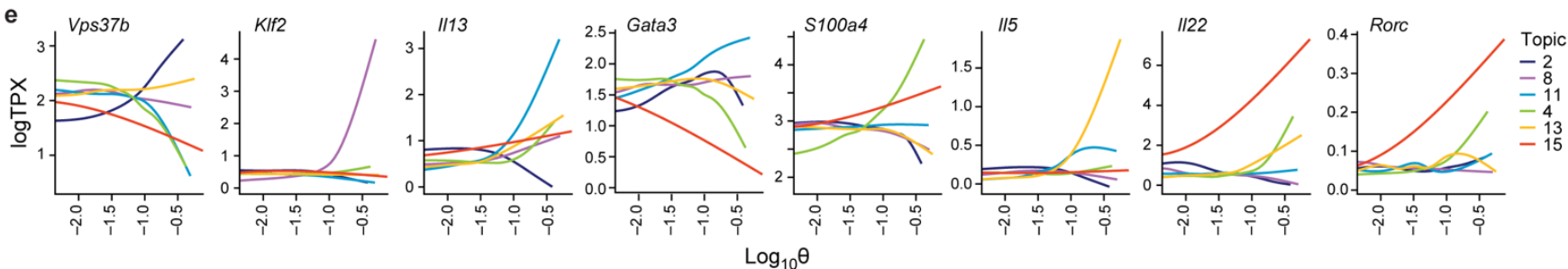


\section{Figure 3. Inference of an IL-23-induced dynamic trajectory from quiescent-like ILCs through classically} activated ILC2s to pathological I13/II17a/Il22-expressing ILC3-like cells. (A,B) Distinct topics suggest a dense continuum of states undergoes a dynamic transition during psoriasis induction. Shown is a diffusion map constructed only from cells highly weighted for selected topics (Topics $2,4,8,11,13$, or 15) and the corresponding topic-specific genes (Methods). Plots of DC2 (x axis), DC3 (y axis) and DC1 (z axis), show cells (dots) colored by either in vivo treatment and genotype (A) or by topic weight $(\log \theta *, j$, color bar) $(\mathbf{B})$. Gray arrows (A) indicate an implicit direction of induction. (C,D) A naïve-induced trajectory across DC1 in a focused diffusion map from Topics 8, 13, and 15. DC 1 (x axis), DC2 (y axis), and DC3 (z axis) of a focused diffusion map, with cells colored as in A by in vivo treatment and genotype (C), or as in B by topic (D). (E), Key genes associated with the trajectory from quiescent-like ILCs to activated ILC2s to ILC3-like cells. Expression (color scale, logTPX) of genes (rows) in cells (columns) associated with Topics 8 (“naïve-quiescent”), 13 (“Il5/Cxcl2"), and 15 (“Il22/Ill17a"), with cells marked by in vivo condition and genotype (top bar; colored as in A). Grey scale bars: Topic weights for cells (log $\left.\theta_{*, j}\right)$ (horizontal bars) and genes (log $\beta_{j, *}$, where $\beta$ is the topic-by-gene weight matrix; vertical bars) illustrate mixtures of functional states. 
bioRxiv preprint doi: https://doi.org/10.1101/461228; this version posted November 12, 2018. The copyright holder for this preprint (which was not certified by peer review) is the author/funder. All rights reserved. No reuse allowed without permission.

\section{Figure 3}

a
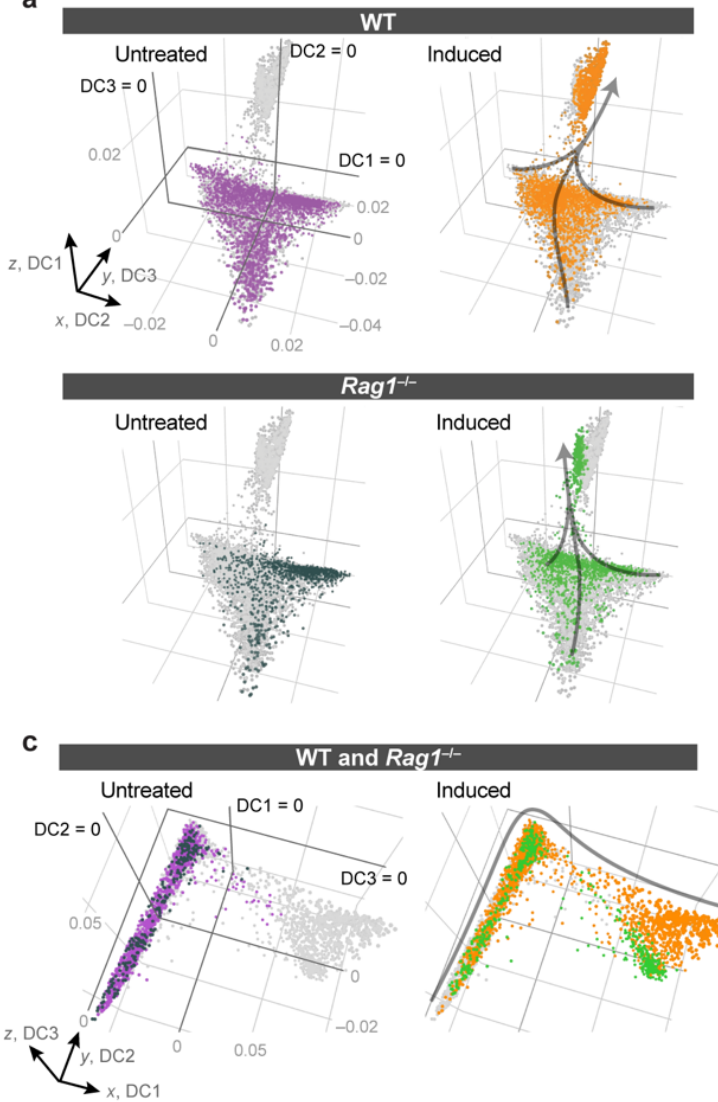

Induced

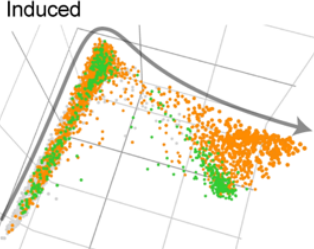

b
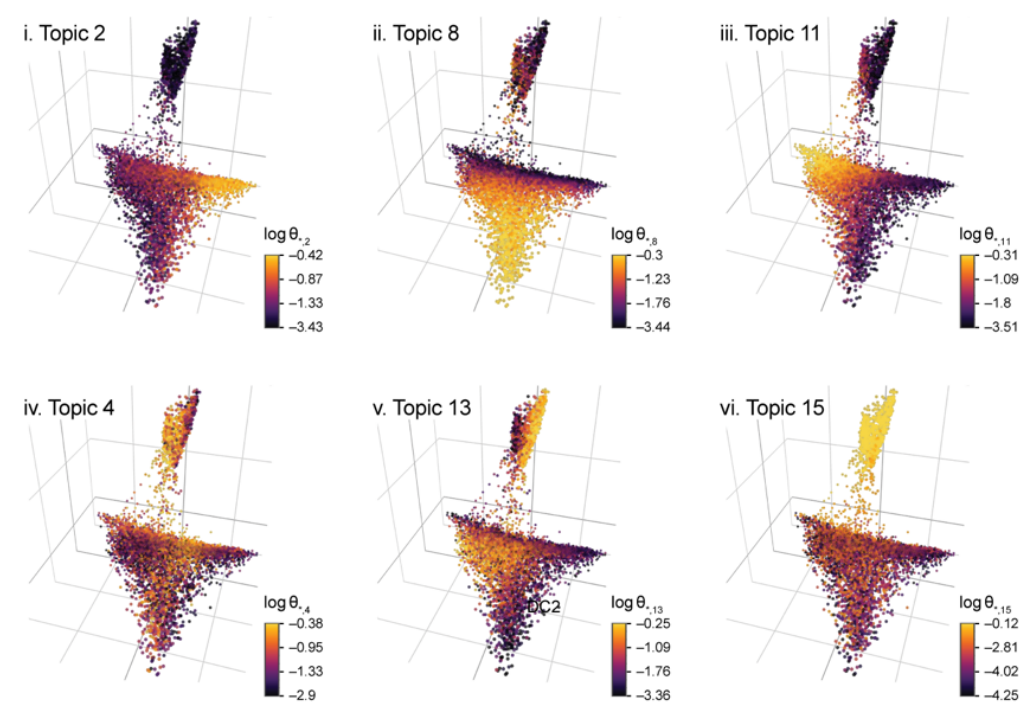

d
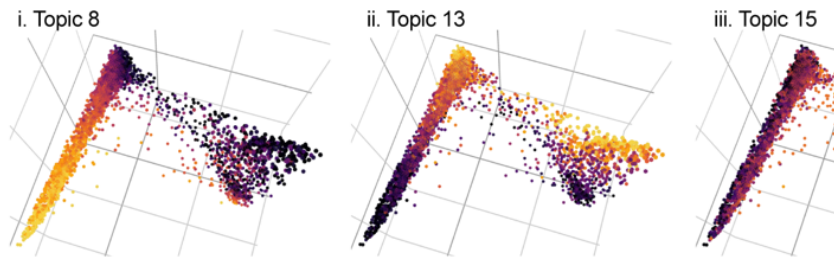

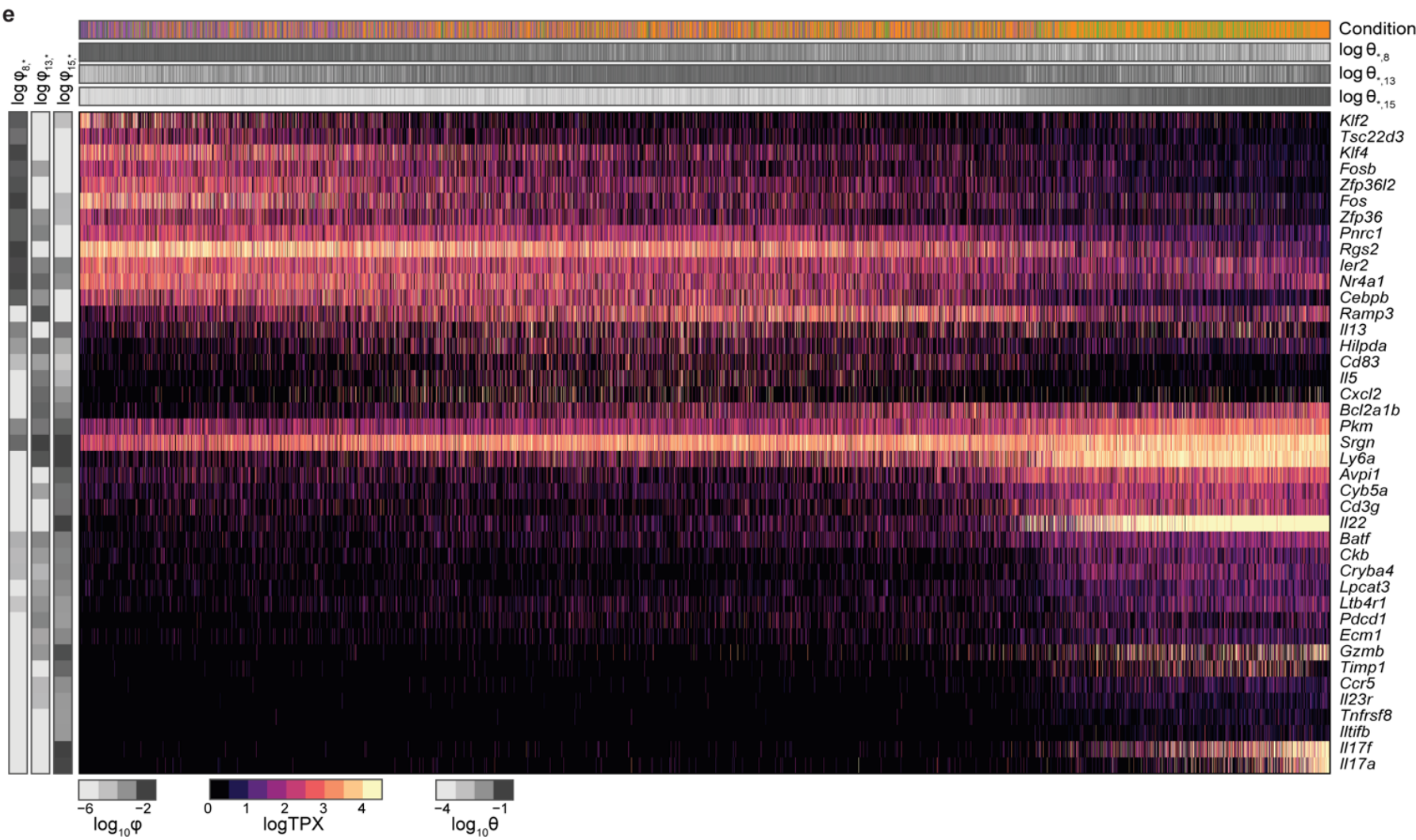


Figure 4. In vivo validation of the trajectory from quiescent-like ILCs in healthy skin to differentiation of ILC2s

to ILC3-like cells during IL-23-induced response. (A) ATAC-seq confirms quiescent-like ILCs. Open chromatin peaks (light blue shaded boxes) of ATAC-Seq reads (blue tracks) from sorted skin ILCs from untreated mice at TSS of key genes (bottom track) responsible for quiescence and repression of type 3 programs. (B-D) ILC2-ILC3 plasticity revealed by IL-5 fate mapping and IL-22 ${ }^{\mathrm{BFP}}$ and IL-17A ${ }^{\mathrm{GFP}}$ reporter mouse. (B) Fate mapping scheme. IL-5 Fate mouse reporter combined with IL-17A ${ }^{\mathrm{GFP}}$ and IL-22 ${ }^{\mathrm{BFP}}$ reporters showing possible outcomes of skin ILC activation, in a scenario with ILC2 to ILC3 differentiation (top) vs. direct ILC3 differentiation (bottom). (C) IL-23 induction increases the number of IL-22- and IL-17A-producing cells, including among cells formerly producing IL-5 (“exIL-5"), especially after secondary challenge. Number of cells (y axis) with each reporter configuration (top label) in IL23-treated and PBS controls (x axis) in the first (circles) and second (squares) challenge. (D) exIL-5 cells that transdifferentiated to produce IL-22 and IL-17A do not produce IL-5 anymore. FACS plots of the expression of YFP (x axis) and IL22-BFP (x axis, top) or IL17A-GFP (x axis, bottom). (E,F), IL-23 treatment induces IL-13/IL-22 and IL-13/IL-17A double-producing populations and elevates IL-13/IL-22 double production in Rag1 deficient mouse. (E) Levels of IL-13 (x axis) and IL22 (y axis) measured by intracellular cytokine staining of skin ILCs in wild type (top), Rag $^{-/-}$(middle) and Tcrd $^{-/}$ (bottom) mice. (F) Mean number of cells (y axis) among single producers and co-producers in each mouse genotype (x axis). Error bars, SD; ${ }^{*} \mathrm{p}<0.0332, * * \mathrm{p}<0.021, * * * \mathrm{p}<0.0002, * * * * \mathrm{p}<0.0001$ by two-way ANOVA. 
bioRxiv preprint doi: https://doi.org/10.1101/461228; this version posted November 12, 2018. The copyright holder for this preprint (which was not certified by peer review) is the author/funder. All rights reserved. No reuse allowed without permission.

\section{Figure 4}
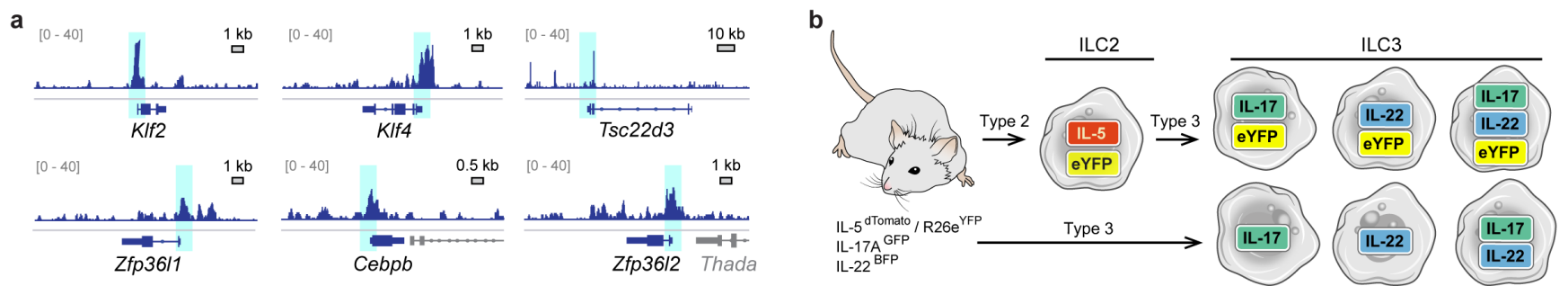

C
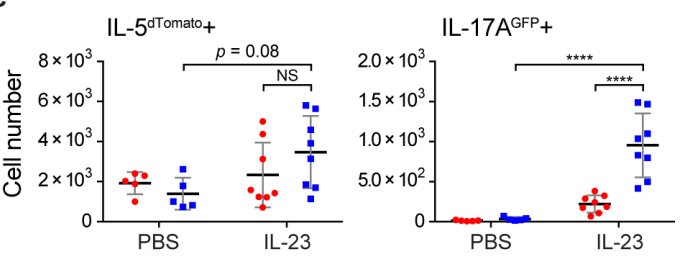

$\mathrm{IL}-22^{\mathrm{BFP}}+$
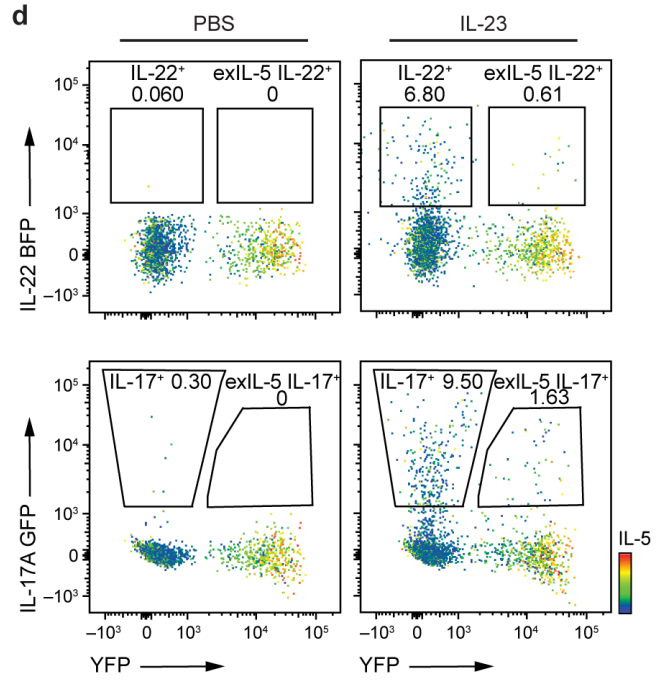

e

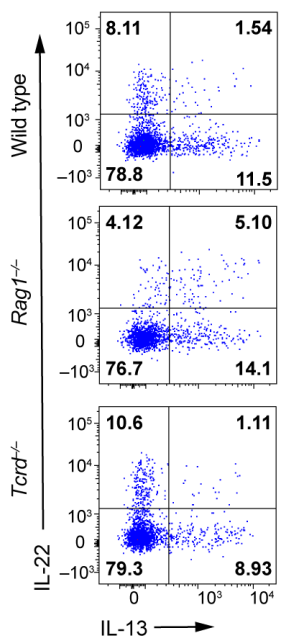

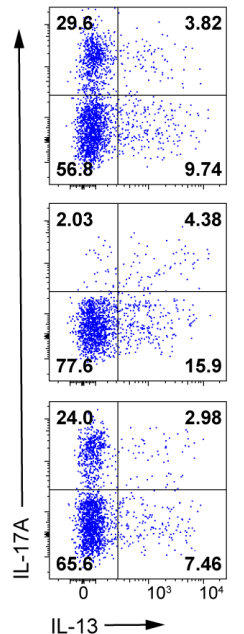
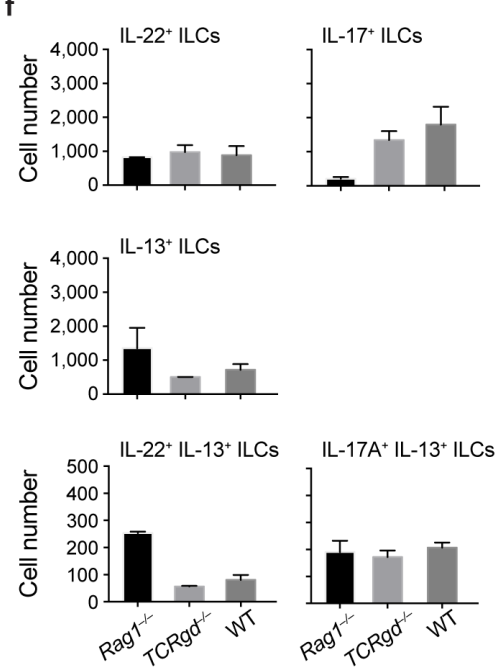


\section{Figure S1. Characterization of skin immune cells to IL-23 induction, Related to Figure 1}

(A) Increase in ear skin thickness is significantly higher in response to IL-23 treatment than PBS vehicle and is dependent on Rorc. Increase in ear thickness (y axis, mm) following treatment with IL-23 (blue) or PBS

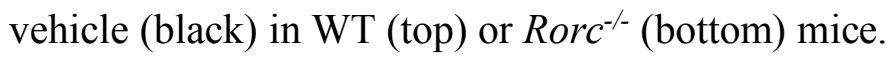

(B and C) Immune cell composition and skin phenotype in different mouse genotypes. (B) Top: Number of cells (y axis) producing IL-22 or IL-17 (x axis) among ILCs (black bars), $\alpha \beta T$ cells (grey bars) and $\gamma \delta \mathrm{T}$ cells

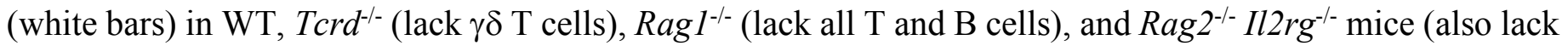
ILCs) mice. Bottom: Number of total CD45 (left, y axis) or total ILCs (right, y axis) in WT, Tcrd ${ }^{-/-}, \operatorname{Rag}^{-/-}$, and $\mathrm{Rag}^{-/-} \mathrm{Il2} \mathrm{rg}^{-/-}$mice (x axis). (C) H\&E stains of ear sections in each genotype except $\mathrm{Rag}^{\mathrm{H}^{-/-}} \mathrm{Il2} \mathrm{rg}^{-/-}$mice. Arrows: Acanthosis.

(D) Expression of type 2 and type 3 related genes in cultured naïve skin ILCs. Shown are relative expression levels (y axis, by qPCR) in ILCs cultured with different cytokines (x axis, table at bottom).

(E) FTY720 treatment does not impact increased susceptibility to a secondary challenge with IL-23. Skin thickness (y axis, $0.1 \mathrm{~mm}$ ) over time ( $\mathrm{x}$ axis, days) in mice following treatment with either IL-23 or IL-23 and FTY720. Bottom bars: period of primary (left bar) and secondary (right bar) challenge.

(F and G) ATAC-seq of sorted skin ILCs from untreated mice. Mapped ATAC-seq reads from sorted skin ILCs from untreated mice (top tracks) at different loci (bottom tracks) of interest. Blue shaded areas: TSS; Beige shared areas: open chromatin peaks at key TF binding sites, previously identified in $\mathrm{CD}^{+} \mathrm{T}$ cell ChIPseq data (middle tracks). (F) Open chromatin peaks at TSS of Gata3 (associated with mature ILC2) but not Rorc (ILC3) and Tbx21 (ILC1). (G) Open chromatin peaks at TF binding sites in the Il17a and Ill $7 f$ promoter (beige), and at the TSS of Il13 but not Il17a,Ill7f(blue).

(H), Cluster B reflects cycling ILCs. tSNE of 27,998 single ILC profiles (dots) colored by expression level (logTPX, color bar) of Stmn1. 
bioRxiv preprint doi: https://doi.org/10.1101/461228; this version posted November 12, 2018. The copyright holder for this preprint (which was not certified by peer review) is the author/funder. All rights reserved. No reuse allowed without permission.

\section{Figure S1}
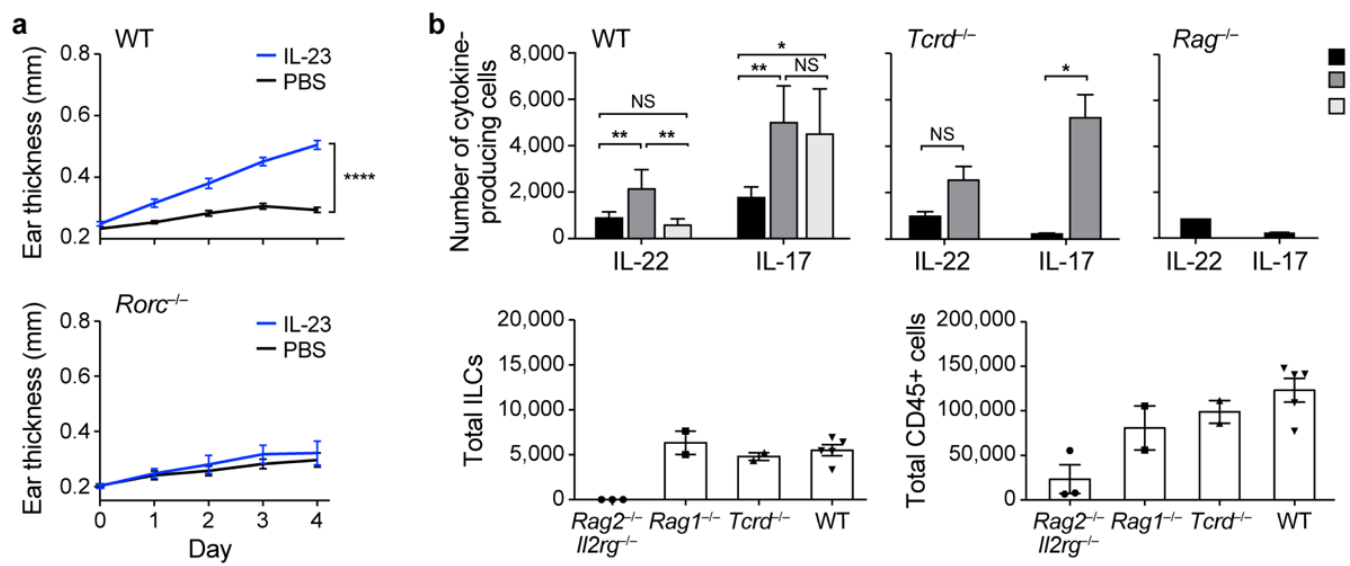

C
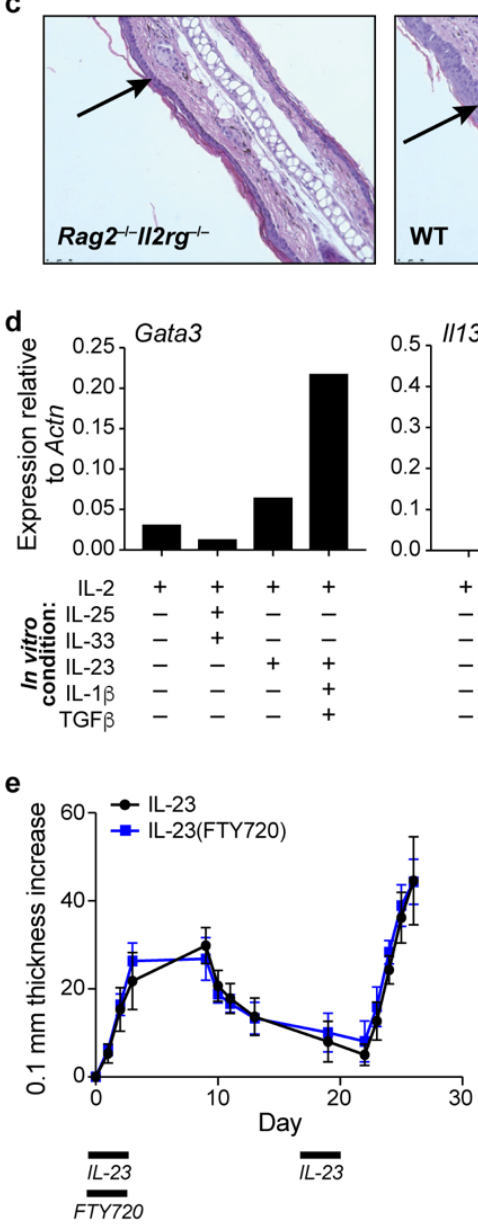

f

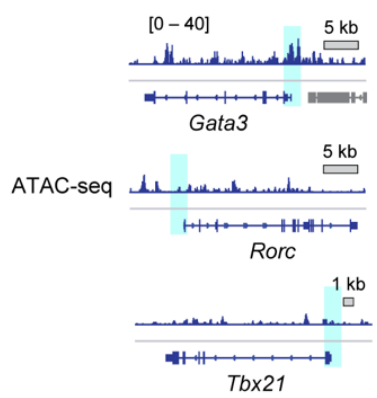

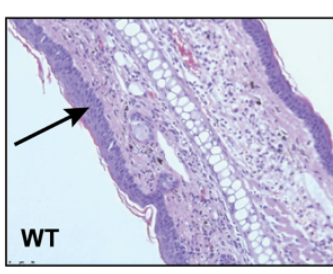
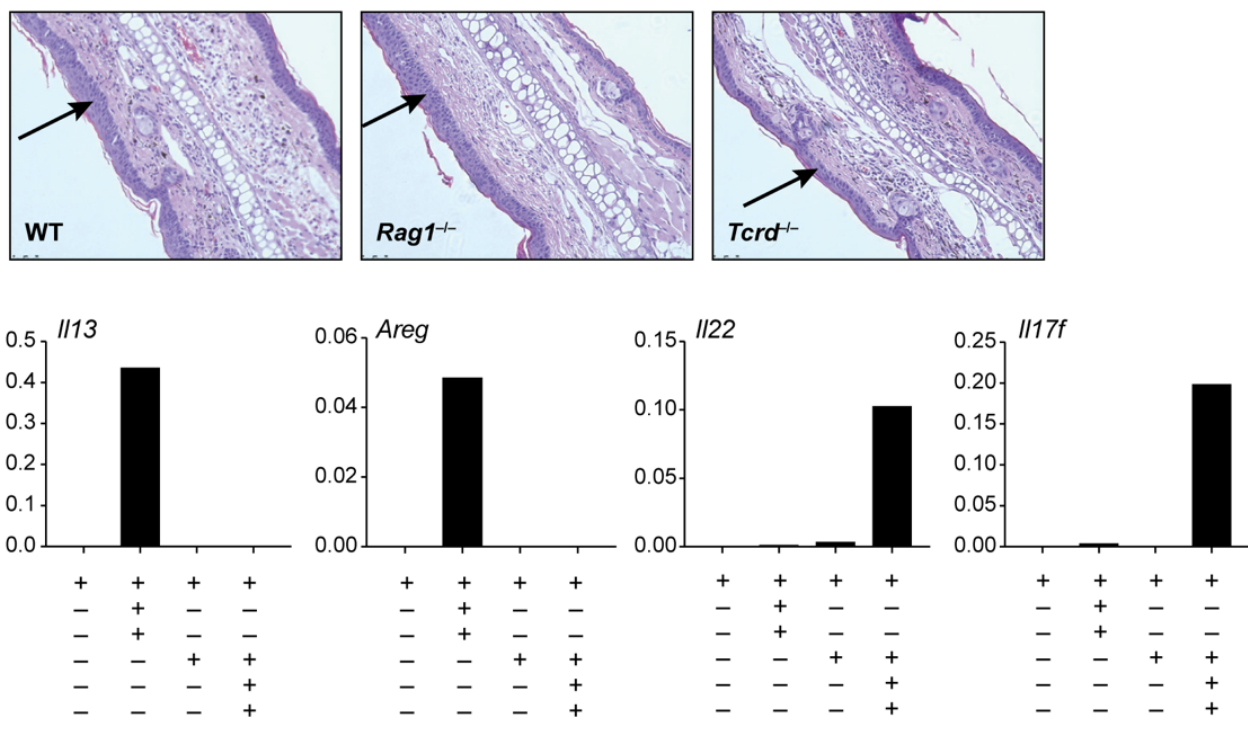

g
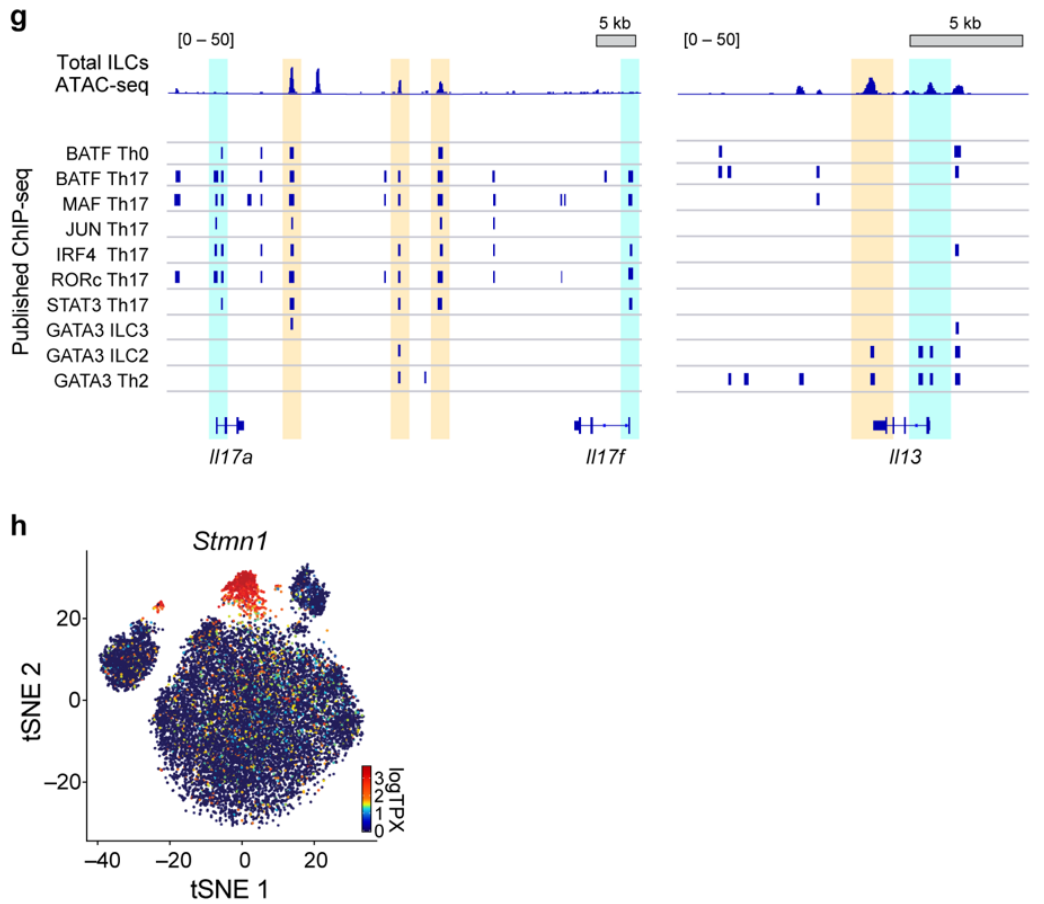
Figure S2. Topic modeling also distinguishes cluster-specific, cell size, and cell quality related topics, Related to Figure 2.

(A) Selecting the number of topics. Akaike Information Criterion (AIC, red) and Bayesian Information Criterion (BIC, blue) value (y axis) for a range of the number $K$ of topics. $K=15$ (dotted line) is at a point where the AIC curve decreases less steeply and the BIC curve begins to rise.

(B and C) Expression of example genes associated with key topics. (B) tSNE of 27,998 single ILC profiles (dots) colored by expression level (color bar, logTPX (Methods)) for genes in Topics 2, 4, 8, 11, 13 , and 15.

(C) Gene expression (y axis, $\log \mathrm{TPX}$ ) as a function of the topic weight $\left(\log \theta_{*}, \mathrm{x}\right.$ axis), for each of these topics (color), for repressive gene Zfp36l2 and activation-associated gene Ly6a.

(D) Summary of remaining topics not included in Fig. 2c. For each topic shown are a bar plot of top scoring genes (y axis), ranked by a score (x axis, logarithmic scale) of how well the gene distinguishes this from other topics (Methods); a tSNE (as in B) with cells colored by the topic's weight in the cells (column $j$ of the cellby-topic weight matrix $\theta(\theta *, j)$ for Topic $j)$; and a graph of the empirical cumulative density function (y axis) of topic weights $\theta *_{, j}$ (x axis) for cells grouped by treatment or genotype (as in Fig. 2c). 
bioRxiv preprint doi: https://doi.org/10.1101/461228; this version posted November 12, 2018. The copyright holder for this preprint (which was not certified by peer review) is the author/funder. All rights reserved. No reuse allowed without permission.

\section{Figure S2}
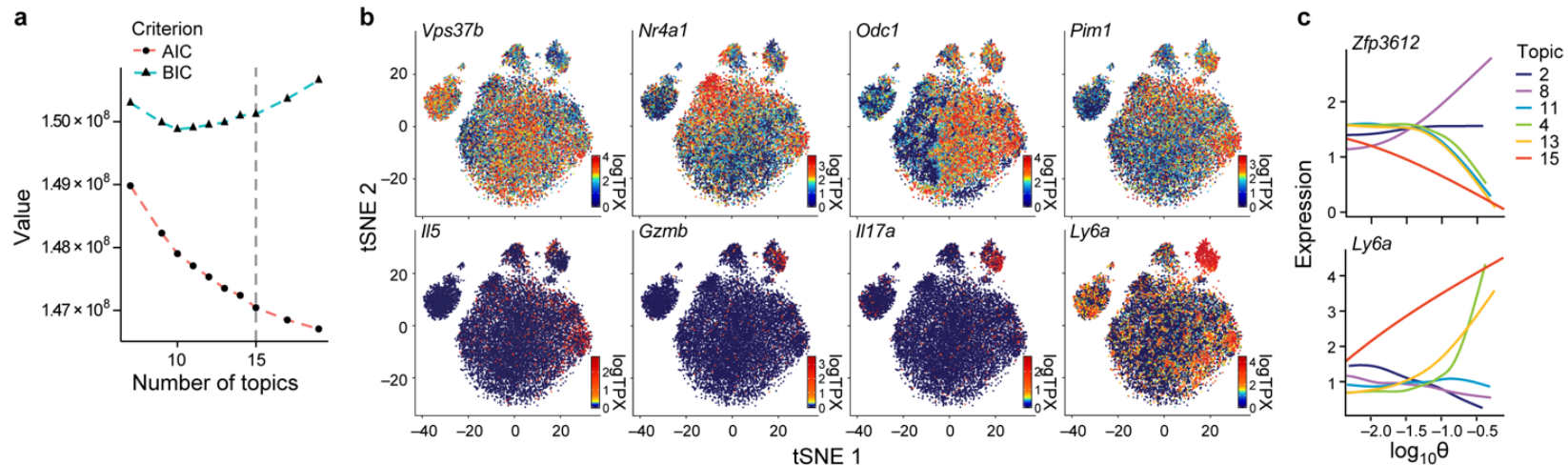

d

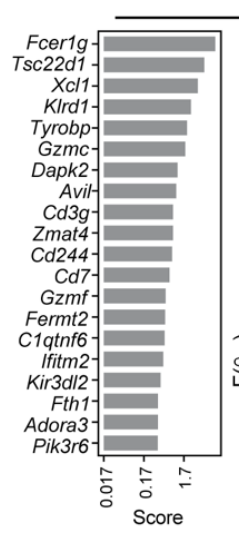

Topic 7

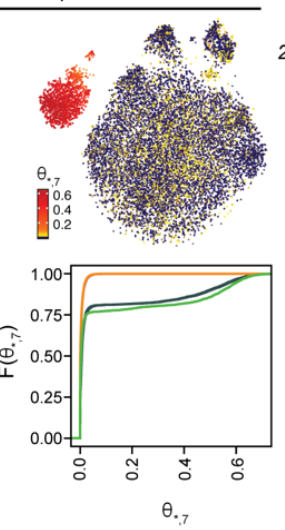

Topic 3
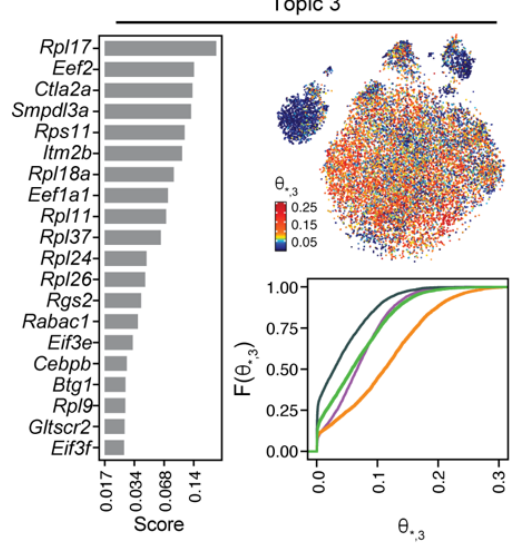

Topic 10

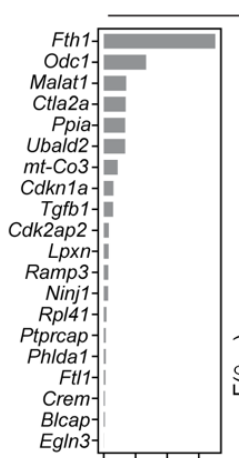

승 둥

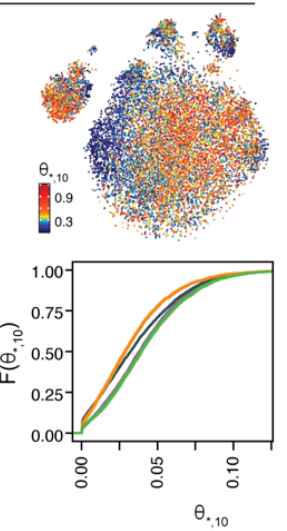

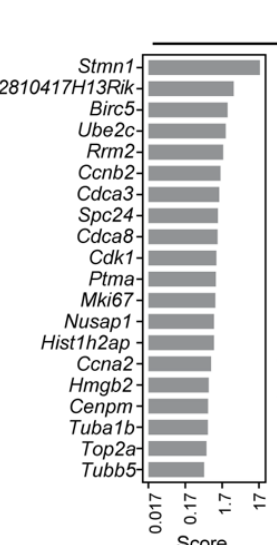

Topic 14
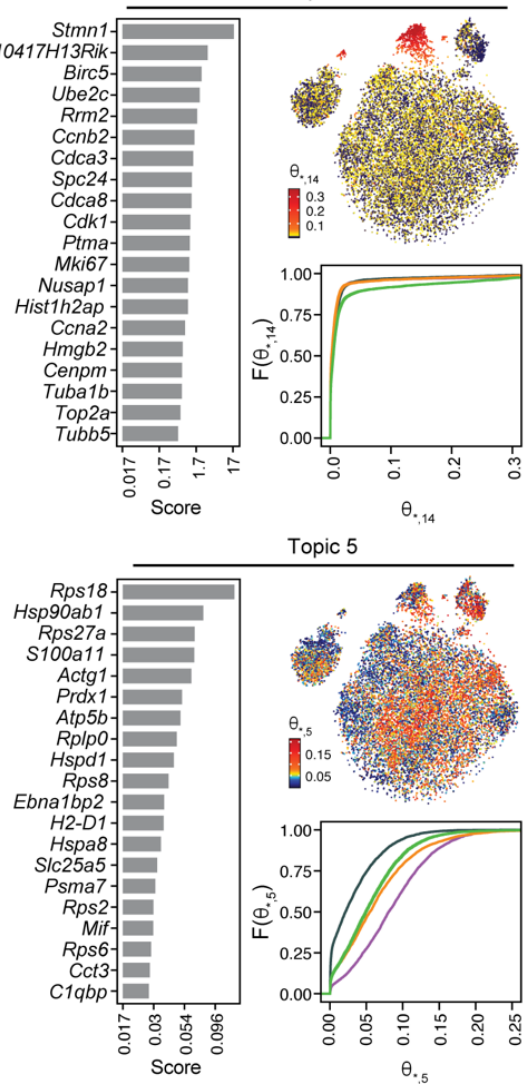

Topic 6
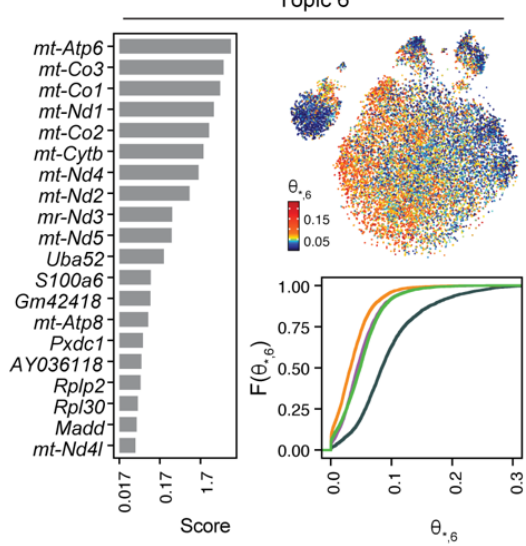

Topic 1

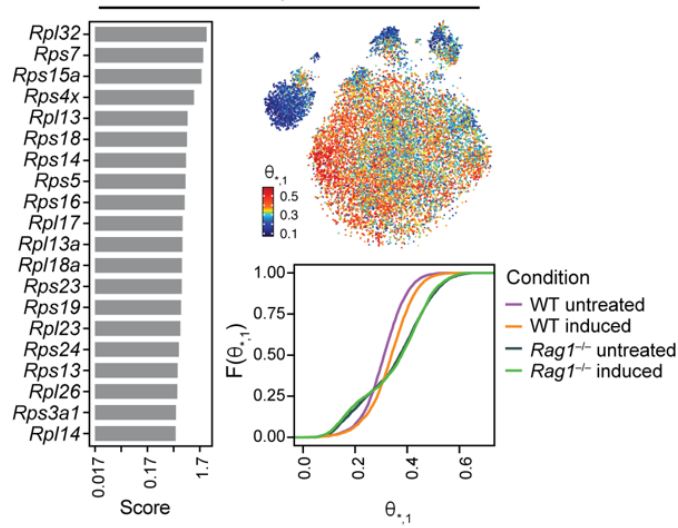

Topic 9

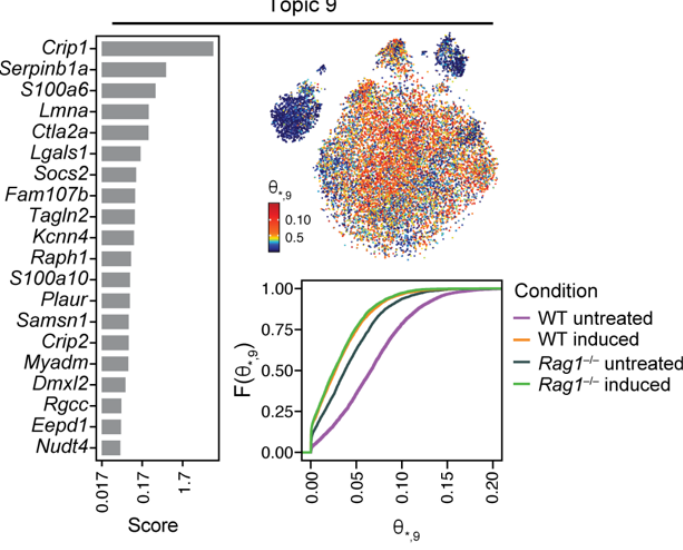

Topic 12
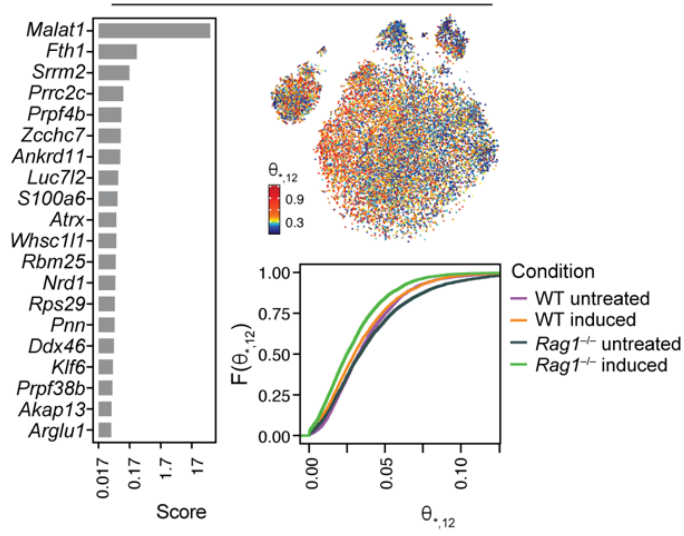
Figure S3. Diffusion map analysis based on topic model highlights an IL-23-induced dynamic trajectory,

\section{Related to Figure 3.}

(A-C) Cell selection for diffusion map in Fig 3A and B. (A) Chosen topic weight thresholds. Empirical cumulative density function (y axis) of topic weights $\theta *_{j}$ ( $\mathrm{x}$ axis) of cells grouped and colored by in vivo treatment and genotype. Dotted line: topic weight threshold. (B and C) Cells with high weights in at least one key topic are chosen for the diffusion map. tSNE of 27,998 single ILC profiles (dots), with cells colored if they are weighted above the corresponding topic threshold from A (B, red) and chosen for the diffusion map (C, black), if they are highly weighted for Topics $2,8,11,4,13$, or 15 , but not for Topics 6 or 7 .

(D and E) Topics 8, 13, and 15 highlight a potential naïve-induced trajectory across DC1. Plots of DC1 (x axis), DC3 (y axis), and DC4 (z axis) show cells (dots) colored by either in vivo treatment and genotype (D) or by topic weight $(\log \theta *, j$, color bar) (E). Gray arrows (D) indicate an implicit direction of induction. 
bioRxiv preprint doi: https://doi.org/10.1101/461228; this version posted November 12, 2018. The copyright holder for this preprint (which was not certified by peer review) is the author/funder. All rights reserved. No reuse allowed without permission.

\section{Figure S3}

a
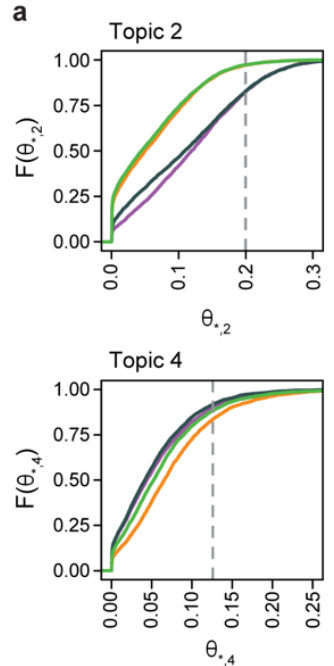

b High Not high

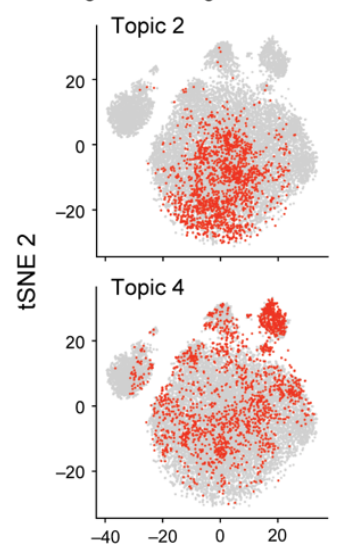

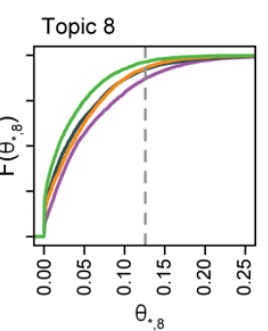
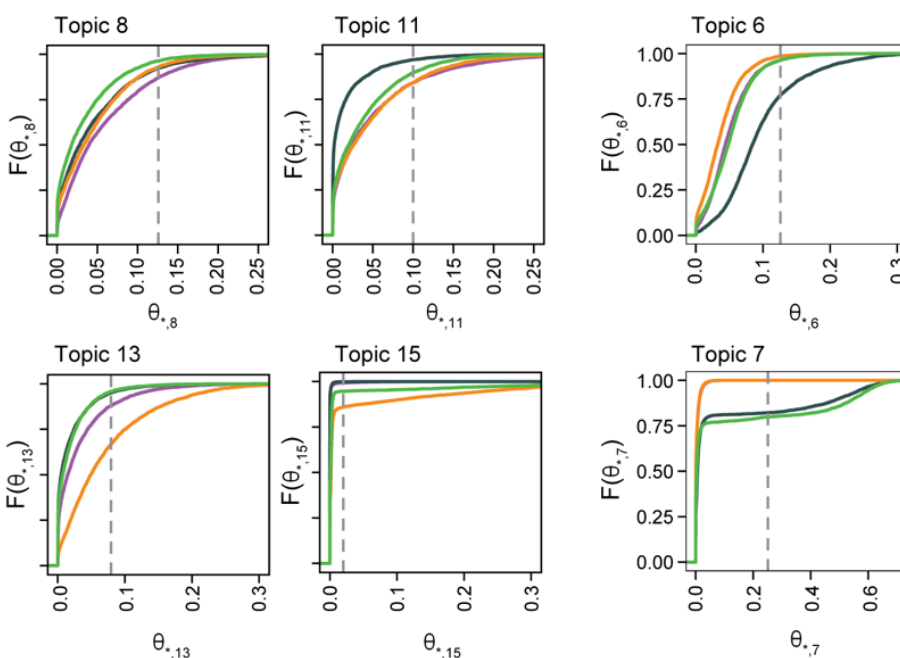

Condition

- WT untreated

- Rag1 $1^{--}$untreated

- Rag 1 ${ }^{-1-}$ induced

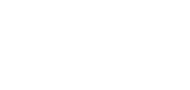

Condition

- WT untreated
- WT induced

- WT induced
- Rag $1^{1-}$ untreated

- Rag 1-- untreated
- Rag $1^{-1-}$ induced

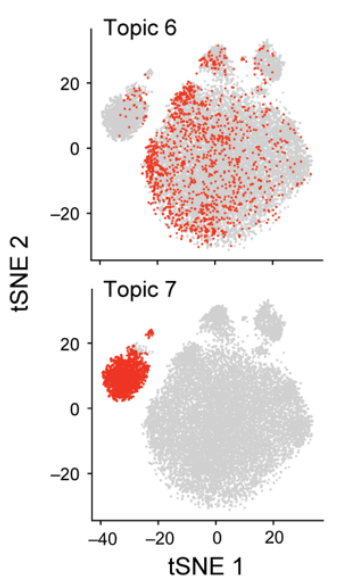

C Included Excluded
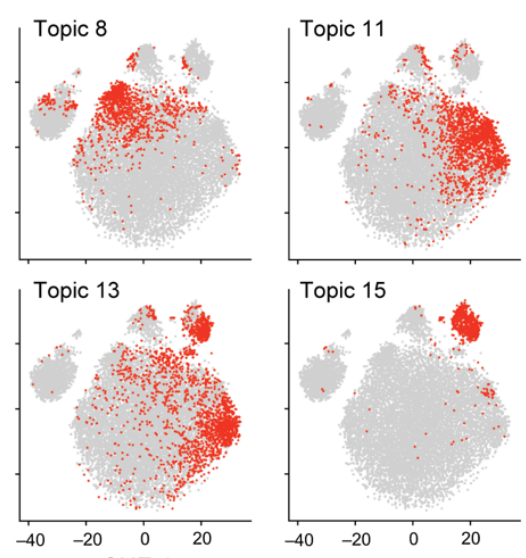

e

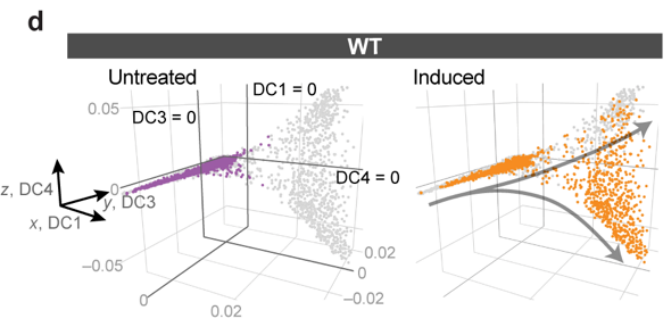

i. Topic 2
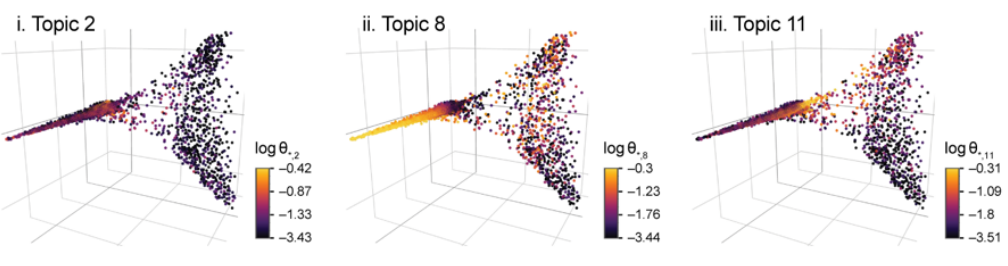

$\operatorname{Rag} 1^{-1}$

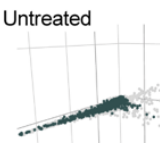

Induced
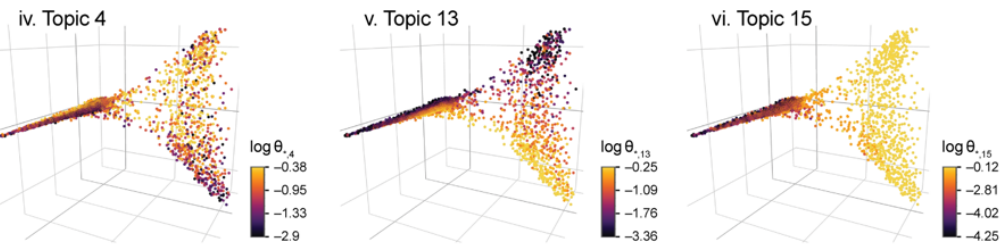


\section{Figure S4. Computational and experimental quality control and data processing.}

(A) IL-23 skin injection model in $I l 5^{\text {dTomatoCre }}($ Red5) mouse strain. Increase in skin thickness (mm, y axis) over time (days, $\mathrm{x}$ axis) in homozygote Red5/Red5 mouse strain lacking expression of IL-5 cytokine (black) and Red5/+ mouse (blue) shown little difference.

(B and C) Quality control and filters in scRNA-Seq. (B) Minimum and maximum thresholds (red) of log UMI counts (x axis) and log gene counts (y axis) for each condition. Cells (dots) that were filtered out are marked in red. (C) Left: Histogram of the \% of mitochondrial genes detected across all cells. Right: Box plot of the \% of mitochondrial genes of all detected genes in each sample type. Dashed lines: thresholds.

(D) Distributions for the number of UMI counts in each sample, used to compute logTPX (Methods).

(E) Variable gene selection. For each sample (panel) shown are the coefficient of variation (CV, y axis) as a function of mean counts (x axis) for each gene (dot). Black curve: null model (Methods). Blue: genes with sufficiently greater CV than in the null model, which are retained as variable genes. 
bioRxiv preprint doi: https://doi.org/10.1101/461228; this version posted November 12, 2018. The copyright holder for this preprint (which was not certified by peer review) is the author/funder. All rights reserved. No reuse allowed without permission.

\section{Figure S4}
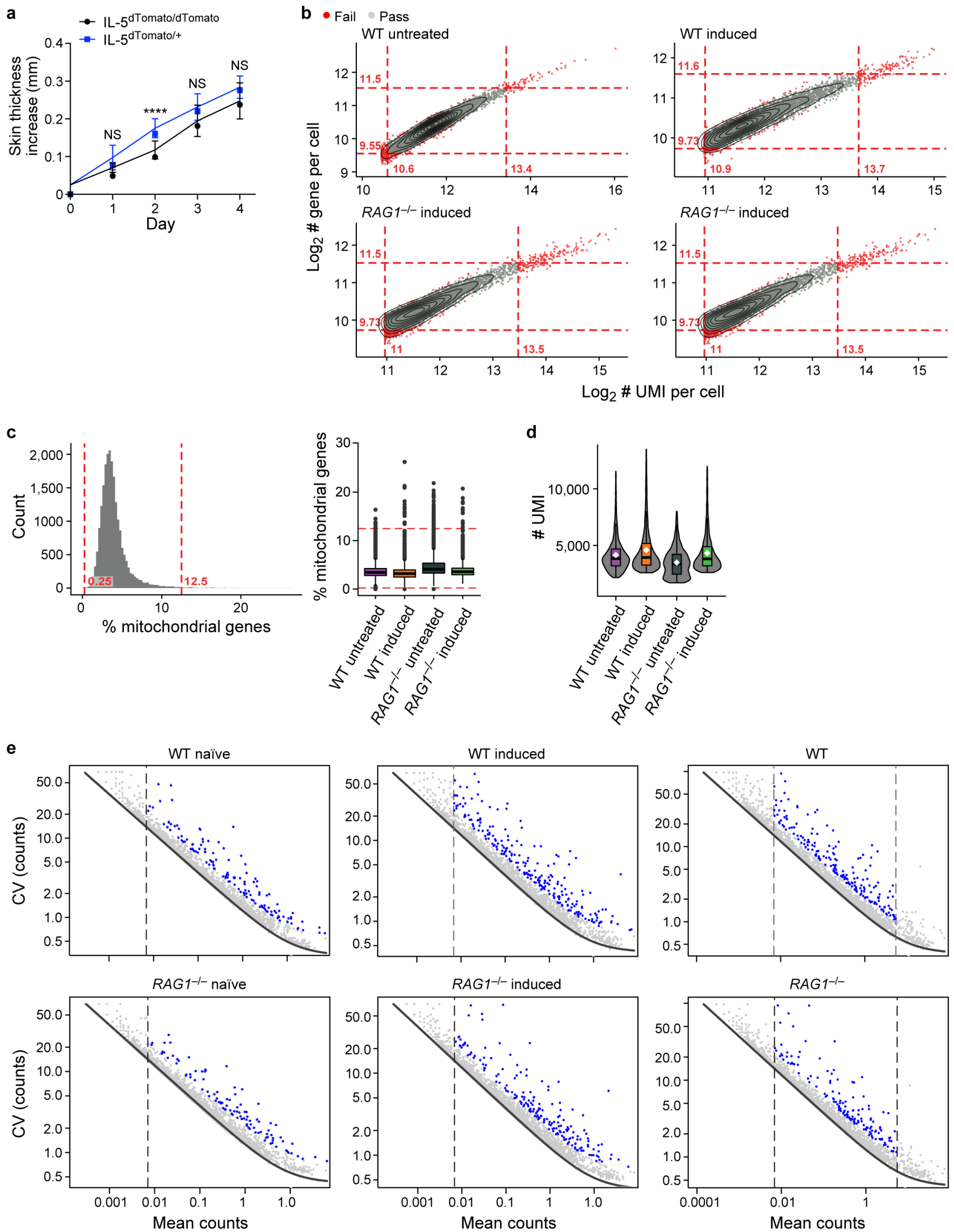